\title{
QUASI-COORDINATES FROM THE POINT OF VIEW OF LIE ALGEBROID STRUCTURES
}

\author{
J.F. CARIÑENA, J.M. NUNES DA COSTA AND PATRÍCIA SANTOS
}

\begin{abstract}
In this paper a geometrical description of the Lagrangian dynamics in quasi-coordinates on the tangent bundle, using the Lie algebroid framework, is given. Linear non-holonomic systems on Lie algebroids are solved in local coordinates adapted to the constraints, through generalized methods of the Lagrangian multipliers and of Gibbs-Appell.
\end{abstract}

KEYWORDS: Lagrangian mechanics, quasi-coordinates, Lie algebroids. AMS Subject Classification (2000): 17B45, 70G10, 70H03, $70 \mathrm{~S} 05$.

\section{Introduction}

The geometric approach to both differential equations and classical mechanics has been very clarifying for many problems and is receiving a lot of attention during the last years. As far as we know there is no systematic treatment of the concept of quasi-coordinate from the geometric point of view, and it is well known that the use of such quasi-coordinates has many applications in physics and engineering [21]. Our aim in this paper is to provide the geometric approach to such a concept and to prove the efficiency of such geometric tool for solving different problems.

The dynamics of a classical system is usually described in terms of generalized coordinates on a tangent bundle of a manifold, i.e. positions $q^{i}$ and their velocities $v^{i} \equiv \dot{q}^{i}$. However, sometimes it is useful to consider a different set of coordinates, e.g. Euler equations for the rigid body are written in terms of the three Euler angles $(\theta, \psi, \varphi)$ and the three components of the angular velocity $\left(\omega_{\theta}, \omega_{\psi}, \omega_{\varphi}\right)$ instead of the velocities $(\dot{\theta}, \dot{\psi}, \dot{\varphi})$. This new set of coordinates are called quasi-velocites. Although usually the calculations in quasi-coordinates are difficult, they are used to solve many types of mechanical systems (see $[14,21]$ ). The geometrical description on the tangent bundle that we propose is a particular case of a more general geometrical framework, the Lagrangian mechanics on Lie algebroids.

Received Julho 13, 2006.

The authors acknowledge the financial support PRODEP/5.3/2003, POCI/MAT/ 58452/2004, CMUC/FCT and the project BFM-2003-02532. 
Lie algebroids provide a very general framework for Mechanics, including mechanical systems with symmetries in the same scheme. Roughly speaking, a Lie algebroid is a generalization of both a Lie algebra and a tangente bundle, these being the simplest examples of Lie algebroids. For a detailed study of this subject we refer the books of Cannas et. al. [2] and of Mackenzie [17]. Since Pradines [22], who introduced the Lie algebroids as infinitesimal objects corresponding to Lie grupoids, several authors have studied the theory of Lie algebroids giving important contributions for the knowledge of their properties and applications. Among others, Higgins et. al. [13] introduced the notion of prolongation of a Lie algebroid over a map; Weinstein [24] was the first to study Lagrangian mechanics on Lie algebroids and Martínez [18] developed the formalism for the Lagrangian mechanics on Lie algebroids, generalizing the fundamental geometrical elements of Lagrangian mechanics (see also [15] and references therein).

One of the goals of this paper is to deal with systems with linear nonholonomic constraints in Lie algebroids, applying a generalized version of the well-known methods of the Lagrangian multipliers [1, 4, 23] and GibbsAppell $[12,16]$. The solution of these systems is obtained in local coordinates adapted to the constraints. These adapted coordinates on a Lie algebroid play the rôle of quasi-coordinates on a tangent bundle. For the subject of non-holonomic systems in a Lie algebroid we refer the first paper from Cortés et. al. [7] and the papers of Mestdag et. al. [19, 20], Cortés et. al. [8] and Cariñena et. al. [6].

This article is organized in the following way. In the first section we recall the geometrical tools of classical mechanics in a tangent bundle. In the second section, we provide the geometric approach to solve classical systems using quasi-coordinates. In the third section we give a brief introduction to Lie algebroids and in the fourth section we see how the geometric approach to quasi-coordinates, given in the second section, derive from a Lie algebroid structure. In the fifth section we generalized the problem of quasi-coordinates in a tangent bundle to the problem of changing coordinates in a Lie algebroid. In the last section we apply the above problem to solve systems with linear non-holonomic constraints in Lie algebroids, using the methods of the Lagrange multipliers and of Gibbs-Appell in Lie algebroid framework. 


\section{The geometric approach to classical mechanics of au- tonomous systems}

In the geometric approach to autonomous systems, the existence of holonomic constraints amounts to restrict the possible configurations of the systems and to consider the set of such possible configurations as a differentiable manifold, usually denoted by $Q$ and called configuration space. Firstorder differential equations appear as local expressions determining the integral curves of vector fields in $Q$, which are sections of the tangent bundle $\tau_{Q}: T Q \rightarrow Q$, while second-order differential equations appear as local expressions determining the integral curves of a special type of vector field on $T Q$, the so-called second-order differential equation vector fields.

A local chart $(U, \phi)$ on the $n$-dimensional manifold $Q$ provides a local trivialization of $\tau_{Q}^{-1}(U)$ and therefore a set of tangent bundle coordinates. If $\left(q^{1}, \ldots, q^{n}\right)$ are the coordinates on $U$, then the set of vector fields on $U$, given by $\left\{\partial / \partial q^{i} \mid i=1, \ldots, n\right\}$, define such a local trivialization and if $v$ is the vector at the point $q$ of coordinates $\left(q^{1}, \ldots, q^{n}\right)$ given by $v=v^{i} \partial / \partial q^{i}$, the local coordinates of $(q, v)$ are $\left(q^{1}, \ldots, q^{n}, v^{1}, \ldots, v^{n}\right)$. Note that $T(T Q)$ has two different vector bundle structures over $T Q$ given, respectively, by $\tau_{T Q}: T(T Q) \rightarrow T Q$ and $T \tau_{Q}: T(T Q) \rightarrow T Q$. Sections with respect to $\tau_{T Q}$ are the vector fields on $T Q$ and those which furthermore are also sections with respect to $T \tau_{Q}$ are the special kind of vector fields to be called second-order differential equation (shortened SODE) vector fields. The local expression of such a SODE vector field is

$$
D=v^{i} \frac{\partial}{\partial q^{i}}+f^{i}(q, v) \frac{\partial}{\partial v^{i}}
$$

The two important geometric ingredients of a tangent bundle are a $(1,1)$ tensor field $S$, called vertical endomorphism, and the Liouville vector field $\Delta$ generating dilations along the fibres (see $[9,10,11]$ ). The coordinate expression of $S$ in terms of natural tangent bundle coordinates $\left(q^{i}, v^{i}\right)$ on $T Q$ is

$$
S=\frac{\partial}{\partial v^{i}} \otimes d q^{i}
$$

while the dilation vector field $\Delta$ on $T Q$, which is the generator of the 1parameter group of dilations $(q, v) \mapsto\left(q, e^{t} v\right)$, is given in such coordinates 
by

$$
\Delta=v^{i} \frac{\partial}{\partial v^{i}}
$$

Both the vector field $\Delta$ and the tensor field $S$ can be used to characterize SODE vector fields $D$, as those satisfying

$$
S(D)=\Delta .
$$

All the constructions in the geometric approach to Lagrangian mechanics can be formulated using such objects $[9,10,11]$. So, given a Lagrangian function $L \in C^{\infty}(T Q)$ we can define the Cartan 1-form $\theta_{L}=d L \circ S$, with coordinate expression $\theta_{L}=\left(\partial L / \partial v^{i}\right) d q^{i}$, and then the Cartan 2-form $\omega_{L}=$ $-d \theta_{L}$ is given by

$$
\omega_{L}=-d \theta_{L}=\frac{\partial^{2} L}{\partial v^{i} \partial q^{j}} d q^{i} \wedge d q^{j}+\frac{\partial^{2} L}{\partial v^{i} \partial v^{j}} d q^{i} \wedge d v^{j} .
$$

The exact 2 -form $\omega_{L}$ is symplectic when the Lagrangian is regular, which in local coordinates means that $\operatorname{det}\left(\partial^{2} L / \partial v^{i} \partial v^{j}\right) \neq 0$. So, when $\omega_{L}$ is symplectic, it establishes a one-to-one correspondence, $\widehat{\omega}_{L}: \mathfrak{X}(T Q) \rightarrow \Omega^{1}(T Q)$, between vector fields and 1-forms on $T Q$ just by contracting vector fields with $\omega_{L}$. In particular, the vector field responsible for the dynamics described by the Lagrangian $L$ in the absence of non-conservative forces, corresponds to the energy function defined by $L, E_{L}=\Delta(L)-L$, and it can be shown to be a SODE vector field.

The remarkable fact is that in such a correspondence, vertical vector fields are in one-to-one correspondence with semi-basic 1-forms on $T Q$ (see [4]). This is an important property because forces arising in classical formulations are geometrically described by semi-basic 1-forms.

The simplest case is when there are not external forces and then the Lagrangian describing the motion is given by the kinetic energy, corresponding to the Riemannian metric $g$ defined on the configuration space $Q$, as a submanifold of the ambient Euclidean space:

$$
T=\frac{1}{2} \widetilde{g}(D, D)
$$

for any choice of a SODE vector field $D$. Here $\widetilde{g}$ denotes the pullback of $g$ to $T Q$. In this case $\theta_{T}(U)=\widetilde{g}(U, \cdot)$, for any $U \in \mathfrak{X}(T Q)$, and $E_{T}=T$. The free dynamics is given by the vector field $X_{T}$, solution of $i\left(X_{T}\right) \omega_{T}-d T=0$. 
The integral curves of $X_{T}$ are the geodesics of the Levi-Civita connection associated with the Riemannian metric $g$ (see [3]).

In the presence of external forces, given by a semibasic 1 -form $\mathcal{F}$ on $T Q$, the dynamical vector field is the solution of

$$
i(X) \omega_{T}-d T=-\mathcal{F},
$$

i.e. $X=X_{T}+\widehat{\omega}_{T}^{-1}(-\mathcal{F})$, where the second term in the sum is vertical and therefore $X$ is also a SODE vector field. Note that $\mathcal{F}$ can also be decomposed into a sum of a non-conservative force $\mathcal{Q}$ with another force which is conservative $\mathcal{F}_{c}$, derivable from potentials, i.e. a (basic) exact form $\mathcal{F}_{c}=-d V$ which can be absorbed in the first term of (1) just by redefining the Lagrangian, now becoming $L=T-V$. In summary, the equations of motion will be

$$
i(X) \omega_{L}-d E_{L}=-\mathcal{Q},
$$

where we assume that the conservative forces are included in the definition of $L$, because $\omega_{L}=\omega_{T}$ and $E_{L}=T+V$. The Lagrangian functions of the form $L=T-V$ are usually said to be of mechanical type.

Now, one can develop the theory for general Lagrangian functions $L \in$ $C^{\infty}(T Q)$, where the conservative forces are included in its definition, with the only assumption of regularity, i.e. it is assumed that $L$ is regular.

\section{A geometric approach to quasi-coordinates}

The use of quasi-coordinates has been shown to be very efficient in describing the motion of some dynamical systems. For instance, the use of the area swept by the line joining a planet with the sun for the motion of the planet, or the use of the components of the angular momentum for describing the motion of a rigid body with a fixed point. In fact, as pointed out in [12, 25], the configuration of a dynamical system cannot be in general described by quasi-coordinates, but it is possible to describe the displacement by using quasi-coordinates, more specifically quasi-velocities. We explain next the geometric meaning of such quasi-velocities.

Let $\pi_{Q}: T^{*} Q \rightarrow Q$ denote the cotangent bundle of a $n$-dimensional differentiable manifold $Q$. It is well-known that a local 1-form $\alpha$ on an open set $U$ of $Q$, i.e. a section for $\pi_{Q}$ over $U$, defines a linear function $\widehat{\alpha} \in C^{\infty}(\mathcal{U})$, where $\mathcal{U}=\tau_{Q}^{-1}(U)$, as follows:

$$
\widehat{\alpha}(v)=\left\langle\alpha_{\tau_{Q}(v)}, v\right\rangle,
$$

for all local vector field $v$ defined on the open set $U$. 
As indicated above, the choice of a local chart on an open set $U$ of $Q$, with coordinates $\left(q^{1}, \ldots, q^{n}\right)$, determines a basis $\left(\partial / \partial q^{1}, \ldots, \partial / \partial q^{n}\right)$ of the tangent space at each point of $\mathcal{U}=\tau_{Q}^{-1}(U)$. In such a way we define an associated local coordinate system on $T Q$. The $2 n$ coordinates are now the $n$ basic functions $q^{i}$ (or in a more rigorous notation $q^{i} \circ \tau_{Q}$ ) and the corresponding functions on the fibres $v^{i}=\widehat{d q^{i}}$. Note that $d q^{1} \wedge \cdots \wedge d q^{n} \neq 0$ and therefore the functions $\widehat{d q^{i}}$, for $i=1, \ldots, n$, are functionally independent.

When the local expression of the local 1-form $\alpha$, in terms of the coordinates on $U$, is $\alpha=\alpha_{i}(q) d q^{i}$, then $\widehat{\alpha}$ is the function $\widehat{\alpha}(v)=\alpha_{i}(q) v^{i}$. In order to define a local chart on $\mathcal{U}$, instead of $\left\{d q^{1}, \ldots, d q^{n}\right\}$, we can alternatively make use, together with the base coordinate functions, of any other set of $n 1$-forms $\left\{\alpha^{1}, \ldots, \alpha^{n}\right\}$, given locally by

$$
\alpha^{i}=\alpha^{i}{ }_{j}(q) d q^{j}, \quad \forall i=1, \ldots, n,
$$

with the only condition of being linearly independent at each point, i.e. $\alpha^{1} \wedge \cdots \wedge \alpha^{n} \neq 0$, in this case the $2 \mathrm{n}$ coordinates in $\mathcal{U}$ are the $\mathrm{n}$ basic functions $q^{i}$ and the linear functions $\left\{\widehat{\alpha^{1}}, \ldots, \widehat{\alpha^{n}}\right\}$. These new coordinates on the fibres $\left\{\widehat{\alpha^{1}}, \ldots, \widehat{\alpha^{n}}\right\}$ are but linear combinations, with basic functions as coefficients, of the usual velocities. Note that the 1 -forms $\alpha^{i}$ do not need to be exact, but in the case of all of them being exact, the functions $\widehat{\alpha^{i}}$ would be the velocities corresponding to a new coordinate system on the base manifold $Q$. The new fibre coordinates $w^{i}=\widehat{\alpha^{i}}$, which play the rôle of velocities, are called quasi-velocities, while the coordinates $\left(q^{i}, w^{i}\right)$ are called quasi-coordinates on $T Q$. Note also that in some cases we can globally define quasi-velocities on $Q$, for instance when $Q$ is a Lie group $G$, while velocities can only be defined locally.

The fact that $\alpha^{1} \wedge \cdots \wedge \alpha^{n} \neq 0$, points out that there exists functions $\beta^{i}{ }_{j}(q)$ such that

$$
d q^{i}=\beta^{i}{ }_{j}(q) \alpha^{j}, \quad \forall i=1, \ldots, n,
$$

with $\operatorname{det}\left(\beta^{i}{ }_{j}\right) \neq 0$. The matrix with entries $\beta^{i}{ }_{j}(q)$ will be the inverse matrix of $\left(\alpha^{i}{ }_{j}(q)\right)$, i.e.

$$
\beta^{i}{ }_{j}(q) \alpha^{j}{ }_{k}(q)=\delta_{i k}
$$

for all $q \in U$. The quasi-velocities $w^{i}$ are associated to a basis of vector fields $\left\{X_{1}, \ldots, X_{n}\right\}$ on $Q$, dual to the basis of 1 -forms $\left\{\alpha^{1}, \ldots, \alpha^{n}\right\}$, that is, 
$\left\langle\alpha^{i}, X_{j}\right\rangle=\delta_{i j}$, so locally $X_{j}=\beta^{i}{ }_{j} \partial / \partial q^{i}$, and

$$
w^{i} X_{i}=v^{j} \frac{\partial}{\partial q^{j}} .
$$

Then, the relations among fibre coordinates are

$$
w^{i}=\alpha^{i}{ }_{j} v^{j}, \quad v^{i}=\beta^{i}{ }_{j} w^{j},
$$

and consequently,

and

$$
\frac{\partial w^{i}}{\partial v^{j}}=\alpha_{j}^{i}, \quad \frac{\partial v^{i}}{\partial w^{j}}=\beta_{j}^{i}
$$

$$
\frac{\partial}{\partial v^{i}}=\alpha^{j}{ }_{i} \frac{\partial}{\partial w^{j}}, \quad \frac{\partial}{\partial w^{i}}=\beta^{j}{ }_{i} \frac{\partial}{\partial v^{j}} .
$$

The local expressions of the Liouville vector field $\Delta$ and the vertical endomorphism $S$ in terms of quasi-coordinates are, respectively, given by

$$
\Delta=v^{i} \frac{\partial}{\partial v^{i}}=\beta_{j}^{i} w^{j} \alpha^{j}{ }_{i} \frac{\partial}{\partial w^{j}}=w^{j} \frac{\partial}{\partial w^{j}},
$$

and

$$
S=\frac{\partial}{\partial v^{i}} \otimes d q^{i}=\alpha^{j}{ }_{i} \frac{\partial}{\partial w^{j}} \otimes d q^{i}=\frac{\partial}{\partial w^{j}} \otimes \alpha^{j} .
$$

The explicit coordinate expressions of a SODE vector field $D \in \mathfrak{X}(T Q)$, which is characterized by $S(D)=\Delta$, in terms of quasi-coordinates is

$$
D=\beta_{k}^{i}(q) w^{k} \frac{\partial}{\partial q^{i}}+f^{i}(q, w) \frac{\partial}{\partial w^{i}},
$$

because if

$$
D=h^{i}(q, w) \frac{\partial}{\partial q^{i}}+f^{i}(q, w) \frac{\partial}{\partial w^{i}},
$$

then

$$
S(D)=\alpha^{j}{ }_{i} h^{i}(q, w) \frac{\partial}{\partial w^{j}},
$$

and therefore $S(D)=\Delta$ if and only if $\alpha^{j}{ }_{i} h^{i}(q, w)=w^{j}$, that is, $h^{i}=\beta^{i}{ }_{j} w^{j}$.

Let us consider a system characterized by a regular Lagrangian function $L \in C^{\infty}(T Q)$ and the action of a non-conservative force $\mathcal{Q}$, locally defined by $\mathcal{Q}(q, v)=Q_{i}(q, v) d q^{i}$. The Cartan 1 -form $\theta_{L}=d L \circ S$ is given in quasicoordinates by

$$
\theta_{L}=\left(\frac{\partial L}{\partial q^{k}} d q^{k}+\frac{\partial L}{\partial w^{i}} d w^{i}\right) \circ\left(\alpha^{j}{ }_{i} \frac{\partial}{\partial w^{j}} \otimes d q^{i}\right)=\alpha^{j}{ }_{i} \frac{\partial L}{\partial w^{j}} d q^{i}=\frac{\partial L}{\partial w^{j}} \alpha^{j},
$$


and consequently, the Cartan 2-form $\omega_{L}$ defined by $\omega_{L}=-\mathrm{d} \theta_{L}$, turns out to be

$$
\begin{aligned}
\omega_{L}= & \frac{1}{2}\left[\left(\frac{\partial \alpha^{k}{ }_{i}}{\partial q^{j}}-\frac{\partial \alpha^{k}{ }_{j}}{\partial q^{i}}\right) \frac{\partial L}{\partial w^{k}}+\alpha^{k}{ }_{i} \frac{\partial^{2} L}{\partial q^{j} \partial w^{k}}-\alpha^{k}{ }_{j} \frac{\partial^{2} L}{\partial q^{i} \partial w^{k}}\right] d q^{i} \wedge d q^{j} \\
& +\alpha^{k}{ }_{i} \frac{\partial^{2} L}{\partial w^{j} \partial w^{k}} d q^{i} \wedge d w^{j},
\end{aligned}
$$

that is,

$\omega_{L}=\frac{1}{2}\left[\gamma_{m l}^{k} \frac{\partial L}{\partial w^{k}}+\beta^{j}{ }_{l} \frac{\partial^{2} L}{\partial q^{j} \partial w^{m}}-\beta^{i}{ }_{m} \frac{\partial^{2} L}{\partial q^{i} \partial w^{l}}\right] \alpha^{m} \wedge \alpha^{l}+\frac{\partial^{2} L}{\partial w^{j} \partial w^{k}} \alpha^{k} \wedge d w^{j}$, where the functions $\gamma_{m l}^{k}$ are given by

$$
\gamma_{m l}^{k}=\beta_{m}^{i} \beta_{l}^{j}\left(\frac{\partial \alpha^{k}{ }_{i}}{\partial q^{j}}-\frac{\partial \alpha^{k}{ }_{j}}{\partial q^{i}}\right)
$$

and are known in the literature by Hamel symbols (see [14, 21]). We can now write the coordinate expression in terms of quasi-coordinates of the dynamical equation $i(X) \omega_{L}=d E_{L}-\mathcal{Q}$, where the energy of the system in the absence of non-conservative forces is given by

$$
E_{L}=\Delta L-L=w^{i} \frac{\partial L}{\partial w^{i}}-L .
$$

It is well-known that when $L$ is regular the dynamics will be given by a second-order differential equation vector field (see [10]), of the form $X=$ $\beta_{m}^{i} w^{m} \partial / \partial q^{i}+f^{m}(q, w) \partial / \partial w^{m}$. The left-hand side of the dynamical equation becomes:

$$
\begin{aligned}
i(X) \omega_{L}= & \left(\gamma_{m l}^{k} \frac{\partial L}{\partial w^{k}}+\beta^{j} l \frac{\partial^{2} L}{\partial q^{j} \partial w^{m}}-\beta^{i}{ }_{m} \frac{\partial^{2} L}{\partial q^{i} \partial w^{l}}\right) w^{m} \alpha^{l} \\
& +w^{m} \frac{\partial^{2} L}{\partial w^{j} \partial w^{m}} d w^{j}-f^{m} \frac{\partial^{2} L}{\partial w^{m} \partial w^{l}} \alpha^{l}
\end{aligned}
$$

while the right hand side is

$$
\mathrm{d} E_{L}-\mathcal{Q}=\left(w^{k} \beta^{i}{ }_{l} \frac{\partial^{2} L}{\partial q^{i} \partial w^{k}}-\beta^{i}{ }_{l} \frac{\partial L}{\partial q^{i}}\right) \alpha^{l}+w^{k} \frac{\partial^{2} L}{\partial w^{j} w^{k}} d w^{j}-\Upsilon_{l} \alpha^{l},
$$

where $\Upsilon_{l}=\beta_{i l} Q_{i}$ is the $l$-component in quasi-coordinates of the external force $\mathcal{Q}$. Therefore,

$$
w^{m} \gamma_{m l}^{k} \frac{\partial L}{\partial w^{k}}-w^{m} \beta^{i}{ }_{m} \frac{\partial^{2} L}{\partial q^{i} \partial w^{l}}-f^{m} \frac{\partial^{2} L}{\partial w^{m} \partial w^{l}}=-\beta^{i}{ }_{l} \frac{\partial L}{\partial q^{i}}-\Upsilon_{l} .
$$


The dynamical equation is equivalent to

$$
£_{X} \theta_{L}=d L+\mathcal{Q},
$$

where $£_{X}$ is the Lie derivative. In the physicians notation, the above equation is equivalent to the following system of generalized Euler-Lagrange equations:

$$
\frac{d}{d t}\left(\frac{\partial L}{\partial w^{l}}\right)=\beta^{j}{ }_{l} \frac{\partial L}{\partial q^{j}}+w^{m} \gamma_{m l}^{k} \frac{\partial L}{\partial w^{k}}+\Upsilon_{l}
$$

Suppose that the Lagrangian is of mechanical type, i.e. $L$ is the difference between the kinetic energy $T$ and the potential energy $V$ of the system. Then, the equations of motion are given by:

$$
\frac{d}{d t}\left(\frac{\partial T}{\partial w^{l}}\right)=\beta^{j} l \frac{\partial T}{\partial q^{j}}+w^{m} \gamma_{m l}^{k} \frac{\partial T}{\partial w^{k}}+\Pi_{l},
$$

where $\Pi_{l}=\beta_{j l} F_{j}$ is the $l$-component in quasi-coordinates of the external force $\mathcal{F}=-d V+\mathcal{Q}($ see $[12,25])$.

\section{Lie algebroids}

The structure of Lie algebroid has been shown to be of a great usefulness in Mechanics from the pioneer paper by Weinstein [24]. In particular Martínez showed in [18] that the Lagrangian theory can be developed directly in the Lie algebroid formalism by using new geometric tools which generalize the vertical endomorphism and the Liouville vector field, but now in a generalization of the tangent bundle of $T Q$.

We summarize in this section the basic concepts and definitions of the theory of Lie algebroids.

Definition 4.1. A Lie algebroid with base $M$ is a vector bundle $\tau_{A}: A \rightarrow M$, together with a Lie algebra structure in the space of its sections given by a Lie product $[\cdot, \cdot]_{A}$, and a vector bundle map over the identity in the base, called anchor, $\rho: A \rightarrow T M$, inducing a map between the corresponding spaces of sections, to be denoted with the same name and symbol, such that:

$$
[v, \varphi w]_{A}=\varphi[v, w]_{A}+(\rho(v) \varphi) w
$$

for any pair of sections for $\tau_{A}, v$ and $w$, and each differentiable function $\varphi$ defined on $M$. 
Let $\left\{q^{i} \mid i=1, \ldots, n\right\}$ be local coordinates in a chart on an open set $U \subset M$, and let $\left\{e_{\alpha} \mid \alpha=1, \ldots, s\right\}$ be a basis of local sections of the bundle $U_{A}=\tau_{A}^{-1}(U) \rightarrow M$. Each local section $v$ is written $v=\mathbf{v}^{\alpha} e_{\alpha}$. The local coordinates of $p \in U_{A}$ are $p=\left(q^{i}, \mathbf{v}^{\alpha}\right)$. In a similar way, the corresponding dual basis $\left\{e^{\alpha} \mid \alpha=1, \ldots, s\right\}$ of local sections on $U_{A^{*}}=\pi_{A}^{-1}(U)$, allows us to define local coordinates $\left(q^{i}, \mu_{\alpha}\right)$ for $\pi_{A^{*}}: A^{*} \rightarrow M$.

The local expressions for the Lie product and the anchor map are (summation on repeated indexes is understood):

$$
\left[e_{\alpha}, e_{\beta}\right]_{A}=c_{\alpha \beta}^{\gamma} e_{\gamma}, \quad \rho\left(e_{\alpha}\right)=\rho_{\alpha}^{i} \frac{\partial}{\partial q^{i}},
$$

where $\alpha, \beta, \gamma=1, \ldots, s$, and $i=1, \ldots, n$. The functions $c_{\alpha \beta}{ }^{\gamma} \in C^{\infty}(U)$ and $\rho_{\alpha}^{i} \in C^{\infty}(U)$ are called structure functions of the Lie algebroid. The conditions for $\rho$ to be a Lie algebra homomorphism are

$$
\rho_{\alpha}^{j} \frac{\partial \rho_{\beta}^{i}}{\partial q^{j}}-\rho_{\beta}^{j} \frac{\partial \rho_{\alpha}^{i}}{\partial q^{j}}=\rho_{\gamma}^{i} c_{\alpha \beta}^{\gamma}, \quad \forall i=1, \ldots, n,
$$

and for the Leibniz condition and the Jacobi identity of the bracket $[\cdot, \cdot]_{A}$, are

$$
\sum_{\operatorname{cycl}(\alpha, \beta, \gamma)}\left(c_{\alpha \beta}{ }^{\nu} c_{\nu \gamma}^{\mu}+\rho_{\gamma}^{i} \frac{\partial c_{\beta \alpha}{ }^{\mu}}{\partial q^{i}}\right)=0, \quad \forall \mu=1, \ldots, s .
$$

These equations are called structure equations of the Lie algebroid.

Examples of Lie algebroids are the tangent bundle of a manifold $M$, with the identity as anchor map and the usual bracket of vector fields, or any integrable subbundle of it, and also a finite-dimensional Lie algebra $\mathfrak{g}$, considered as a vector bundle over a point, for which the anchor vanishes identically and the bracket is that of $\mathfrak{g}$. In the first case, with the usual choice of coordinates $\left(q^{i}, v^{i}\right)$ on $A=T M$, induced from local coordinates $\left(q^{i}\right)$ on the base $M$, the structure functions are

$$
c_{i j}{ }^{k}=0, \quad \rho_{j}^{i}=\delta_{i j} .
$$

However, in arbitrary coordinates, the structure functions $c_{i j}{ }^{k}$, in general, do not vanish. For the case of the Lie algebra $\mathfrak{g}$ the structure functions are the structure constants of the Lie algebra $c_{\alpha \beta}{ }^{\gamma}$ and $\rho_{\alpha}^{i}=0$.

Given a Lie algebroid $\left(A, \rho,[\cdot, \cdot]_{A}\right)$ over $M$, there exists a derivation $d_{A}$ of degree one of the graded exterior algebra of forms of $A, \Omega^{\bullet}(A)$, to be called $A$-forms, which is nilpotent of order two, i.e. $d_{A}^{2}=0$ (see e.g. $[2,17]$ ). It 
is called the exterior derivative of the Lie algebroid. In the particular cases of the tangent bundle $T M$ and that of a Lie algebra $\mathfrak{g}, d_{A}$ reduces to the de Rham operator $d$ on the manifold $M$ and the Chevalley differential $d_{\mathfrak{g}}$, respectively.

\section{Quasi-coordinates and the tangent bundle as a Lie algebroid}

When we use quasi-coordinates $\left(q^{i}, w^{i}\right)$ on the tangent bundle $T Q$, then we have an associated choice of a local basis of sections for $\tau_{T Q}: T(T Q) \rightarrow$ $T Q$, i.e. a local basis of vector fields in $T Q$. Such basis is $\left\{X_{j}, \partial / \partial w^{j}\right.$ $j=1, \ldots, n\}$, where $\left\{q_{i} \mid i=1, \ldots, n\right\}$ is a set of local coordinates on $Q$ and $X_{j}=\beta^{i}{ }_{j} \partial / \partial q^{i}$ is an element of the dual basis of $\left\{\alpha^{i} \mid i=1, \ldots, n\right\}$.

Using such a local basis, the relations defining the structure functions of the Lie algebroid $\tau_{T Q}: T(T Q) \rightarrow T Q$ are:

$$
\begin{aligned}
{\left[X_{r}, X_{l}\right] } & =\gamma_{r l}^{m} X_{m}, \quad\left[X_{m}, \frac{\partial}{\partial w^{k}}\right]=\beta_{j m} \frac{\partial \beta_{r k}}{\partial q^{j}} \alpha_{l r} \frac{\partial}{\partial w^{l}}, \quad\left[\frac{\partial}{\partial w^{i}}, \frac{\partial}{\partial w^{j}}\right]=0, \\
\rho\left(X_{m}\right) & =X_{m}, \quad \rho\left(\frac{\partial}{\partial w^{j}}\right)=\frac{\partial}{\partial w^{j}} .
\end{aligned}
$$

Recall that the anchor map $\rho$ on the Lie algebroid $T(T Q)$ is the identity map on $T(T Q)$, the bracket $[\cdot, \cdot]$ on the sections of the Lie algebroid is the usual bracket of vector fields on $T Q$ and $\gamma_{r l}^{m}$ are the Hamel symbols associated with the definition of the quasi-coordinates.

The expression of the exterior differential of the Lie algebroid will be determined, for all $F \in C^{\infty}(T Q)$ by

$$
d F=X_{i}(F) \alpha^{i}+\frac{\partial F}{\partial w^{i}} d w^{i} .
$$

Given a Lagrangian function $L \in C^{\infty}(T Q)$, we can easily calculated its differential, the differential of $E_{L}=\Delta(L)-L$ and the Cartan forms $\theta_{L}=$ $d L \circ S$ and $\omega_{L}=-d \theta_{L}$. Their local expressions in quasi-coordinates are given in Section 3. If the Lagrangian is regular and a non-conservative force $\mathcal{Q}$ is given, the dynamics equation $i(X) \omega_{L}=d E_{L}-\mathcal{Q}$ has a unique solution $X=w^{m} X_{m}+f^{m} \partial / \partial w^{m}$, that satisfies the generalized Euler-Lagrangian equations (3).

Example 5.1. Let us consider a particle $P$ (mass $=1)$ moving in a plane under the action of a force of magnitude $F(r)$ on the direction of a fixed point 
$O$, where $r$ represents the distance between the point $O$ and the particle $P$. Let $\theta$ be the angle that the line $O P$ makes with a fixed direction in the plane, and $\dot{A}$ the area swept by unity of time by the line. The configuration space of the system is $Q=\mathbb{R}^{2}-\{0\}$ and the usual coordinates on the phase space $T Q$ are $q^{1}=r, q^{2}=\theta, \dot{r}, \dot{\theta}$. In order to solve the equations of motion we use the following set of quasi-velocities on $T Q: w^{1}=\dot{r}, w^{2}=2 \dot{A}=r^{2} \dot{\theta}$. From the previous relations, we conclude that the coordinates transformation matrices $\alpha$ and $\beta$ are the following:

$$
\alpha=\left(\alpha^{i}{ }_{j}\right)=\left(\begin{array}{cc}
1 & 0 \\
0 & r^{2}
\end{array}\right), \quad \beta=\left(\beta_{j}^{i}{ }_{j}\right)=\left(\begin{array}{cc}
1 & 0 \\
0 & \frac{1}{r^{2}}
\end{array}\right) .
$$

The motion of the particle $P$ is described by the regular Lagrangian

$$
L=\frac{1}{2}\left(w^{1}\right)^{2}+\frac{1}{2} \frac{\left(w^{2}\right)^{2}}{r^{2}}-V(r),
$$

where $F_{1}=-d V / d r=F(r)$ and $F_{2}=0$. The equations of motion (4) are equivalent to

$$
\left\{\begin{array}{l}
\Pi_{1}\left(r, \theta, w^{1}, w^{2}\right)=\dot{w^{1}}-\frac{\left(w^{2}\right)^{2}}{r^{3}} \\
\Pi_{2}\left(r, \theta, w^{1}, w^{2}\right)=\frac{\dot{w}^{2}}{r^{2}}
\end{array} .\right.
$$

Since $\Pi_{1}=\beta^{1}{ }_{1} F_{1}+\beta^{2}{ }_{1} F_{2}=F_{1}$ and $\Pi_{2}=\beta^{1}{ }_{2} F_{1}+\beta^{2}{ }_{2} F_{2}=0$, the dynamics is given by the integrable curves of the vector field $X=w^{m} X_{m}+f^{i} \partial / \partial w^{i}$ satisfying:

$$
\left\{\begin{array}{l}
\dot{w^{1}}=F(r)+\frac{\left(w^{2}\right)^{2}}{r^{3}}=f^{1} \\
\dot{w^{2}}=0=f^{2}
\end{array}\right.
$$

where $\dot{r}=w^{1}$ and $\dot{\theta}=w^{2} / r^{2}$. As we can easily see, the quasi-velocity $w^{2}$ is a constant of motion, that is, the area swept $\dot{A}$ is constant of motion.

The usual coordinates on TQ are: $q^{1}=r, q^{2}=\theta, v^{1}=\dot{r}$ and $v^{2}=\dot{\theta}$. If we need to determine the geometrical solution of the dynamics in the usual set of coordinates on $T Q$, we must pay attention to the term with $\partial / \partial q^{i}$ in the solution $X$ in quasi-coordinates, that is, the parcial derivative in order to the coordinate $q^{i}$ maintaining fixed the coordinates $w$ :

$$
\left.\frac{\partial}{\partial q^{i}}\right|_{w}=\left.\frac{\partial}{\partial q^{i}}\right|_{v}+\frac{\partial}{\partial v^{j}} \frac{\partial v^{j}}{\partial q^{i}}=\left.\frac{\partial}{\partial q^{i}}\right|_{v}+\frac{\partial}{\partial v^{j}} \frac{\partial \beta^{j} k}{\partial q^{i}} \alpha^{k}{ }_{r} v^{r}
$$


QUASI-COORDINATES FROM THE POINT OF VIEW OF LIE ALGEBROID STRUCTURES 13

In this particular case,

$$
X=w^{m} X_{m}+\left(F\left(q^{1}\right)+\frac{\left(w^{2}\right)^{2}}{\left(q^{1}\right)^{3}}\right) \frac{\partial}{\partial w^{1}}=\left.w^{m} \beta_{m}^{i} \frac{\partial}{\partial q^{i}}\right|_{w}+\left(F\left(q^{1}\right)+\frac{\left(w^{2}\right)^{2}}{\left(q^{1}\right)^{3}}\right) \frac{\partial}{\partial w^{1}} .
$$

So, applying (8), we have the dynamics solution in usual coordinates $\left(q^{1}, q^{2}\right.$, $\left.v^{1}, v^{2}\right)$ on $T Q$

$$
X=\left.v^{i} \frac{\partial}{\partial q^{i}}\right|_{v}+v^{i} \frac{\partial}{\partial v^{j}} \frac{\partial \beta^{j} k}{\partial q^{i}} \alpha_{r}^{k} v^{r}+\left(F\left(q^{1}\right)+\frac{\left(w^{2}\right)^{2}}{\left(q^{1}\right)^{3}}\right) \beta^{i}{ }_{1} \frac{\partial}{\partial v^{i}},
$$

that is,

$$
X=\left.v^{i} \frac{\partial}{\partial q^{i}}\right|_{v}+v^{1} \frac{\partial}{\partial v^{2}}\left(\frac{-2}{r^{3}}\right) r^{2} v^{2}+\left(F\left(q^{1}\right)+\frac{\left(w^{2}\right)^{2}}{\left(q^{1}\right)^{3}}\right) \frac{\partial}{\partial v^{1}} .
$$

Then, in the coordinates $(r, \theta, \dot{r}, \dot{\theta})$ we have

$$
X=\dot{r} \frac{\partial}{\partial r}+\dot{\theta} \frac{\partial}{\partial \theta}+\left(F(r)+r \dot{\theta}^{2}\right) \frac{\partial}{\partial \dot{r}}-\frac{2 \dot{r} \dot{\theta}}{r} \frac{\partial}{\partial \dot{\theta}} .
$$

Example 5.2. Let $G$ be a Lie group and e the unity of the group. We can identify the tangent bundle $T G$ with $G \times T_{e} G$, using the map $T L_{g^{-1}}: T G \rightarrow$ $G \times T_{e} G$ given by

$$
T L_{g^{-1}}(g, \dot{g})=(g, \xi),
$$

where $L_{g}: G \rightarrow G$ is defined by $L_{g} h=g h$, for all $h \in G$. Let $\left(\xi^{I}\right)$, for $I=1, \ldots, \operatorname{dim} G$, be the set of coordinates of $\xi \in T_{e} G$ with respect to a base $\left\{e_{I}\right\}$ of $T_{e} G$, then $\left(\xi^{I}\right)$ is a set of quasi-velocities in $T G$, where

$$
\xi^{I} e_{I}=\xi=T_{g} L_{g^{-1}}(\dot{g}) .
$$

If $g$ is a point in $G$ of local coordinates $\left(g^{I}\right)$, then $\left(g^{I}, \xi^{I}\right)$ define a set of quasi-coordinates in TG. Note that the map $\alpha=T_{g} L_{g^{-1}}: T_{g} G \rightarrow T_{e} G$ is an invertible linear transformation whose inverse transformation is given by $\beta=T_{e} L_{g}: T_{e} G \rightarrow T_{g} G$. Thus, $\xi^{I}=\alpha^{I}{ }_{J} \dot{g}^{J}$ and $\dot{g}^{I}=\beta^{I}{ }_{J} \xi^{J}$, where $\alpha=$ $\left(\alpha_{J}^{I}\right)$ and $\beta=\left(\beta_{J}^{I}\right)$ are the coordinates transformations matrices, between the usual coordinates $\left(g^{I}, \dot{g}^{I}\right)$ on TG and the quasi-coordinates $\left(g^{I}, \xi^{I}\right)$.

Given a regular and $G$-invariant Lagrangian $L \in C^{\infty}(T G)$, it does not depend on the elements of the Lie group $G$. Thus, in quasi-coordinates, the Lagrangian is given by $l(\xi)=L_{q c}(g, \xi)=L(g, \dot{g})$. Since the Lagrangian $L$ 
on the Lie algebroid $T G$ is G-invariant, it is possible to obtain the EulerLagrange equations of the gauge algebroid $T G / G \equiv \mathfrak{g}$ from the equations (3) on $T G$ :

$$
\frac{d}{d t}\left(\frac{\partial l}{\partial \xi^{I}}\right)=\xi^{J} c_{J I}^{K} \frac{\partial l}{\partial \xi^{K}}
$$

where $c_{J I}{ }^{K}$ are the structure constants of the Lie algebra $\mathfrak{g}$ of the Lie group $G$, with respect to the basis $\left\{e_{I}\right\}$ of $\mathfrak{g} \equiv T_{e} G$. In fact, let $X^{L}(g)=T_{e} L_{g}(X)$ be the left-invariante vector field on $G$ associated to an element $X$ in the Lie algebra $\mathfrak{g}$ of $G$. Thus, the set $\left\{e_{I}^{L}\right\}$ represents a local basis of sections of $T G$ associated to the set of local coordinates $\left(g^{I}, \xi^{I}\right)$ on $T G \equiv G \times \mathfrak{g}$, because

$$
\xi^{I} e_{I}^{L}=\dot{g}^{i} \frac{\partial}{\partial g^{i}}
$$

The structure functions of the Lie algebroid TG are given by:

$$
\left[e_{I}^{L}, e_{J}^{L}\right]_{T G}=c_{I J}{ }^{K} e_{K}^{L}, \quad \rho_{T G}\left(e_{J}^{L}\right)=e_{J}^{L} .
$$

Using the structure functions of the Lie algebroid TG and the Euler-Lagrangian equations (3), in the absence of non-conservative forces, we obtain (9). If the Lagrangian $L$ is regular, then the geometric solution of the dynamics is a SODE vector field on TG given by $X_{L}=\xi^{M} e_{M}^{L}+f^{M} \partial / \partial \xi^{M}$ with

$$
f^{M}=\bar{W}^{M I} \xi^{J} c_{J I}{ }^{K} \frac{\partial l}{\partial \xi^{K}},
$$

where $\bar{W}^{M I}$ is the inverse matriz of $\left(\partial^{2} l / \partial \xi^{I} \partial \xi^{M}\right)$.

\section{The problem of changing local coordinates on a Lie algebroid with base coordinates fixed}

Using the description of Lagrangian mechanics in a Lie algebroid (see [15, 18]) and the structure functions of the Lie algebroid for a given set of local coordinates, it is possible to write the dynamics of the Lie algebroid in a different set of coordinates, in parallel with quasi-coordinates formalism on a tangent bundle (see $[12,14]$ ). In an arbitrary Lie algebroid, we do not have a canonical basis of sections for the Lie algebroid and, therefore, the Lie algebroid does not have a natural set of coordinates. However, sometimes is worthwhile to write the equations of motion in a given set instead of other initially given, as we can see in the next section. 
Recall that the prolongation of the Lie algebroid $p: A \rightarrow M$ (see $[13,15$, 18]) is a vector bundle $\mathcal{T} A$ over $A$, where $\mathcal{T} A$ is the total space of the pullback of the vector bundle $T p: T A \rightarrow T M$ by the anchor map $\rho: A \rightarrow T M$. The projection $p_{\mathcal{T} A}: \mathcal{T} A \rightarrow A$ is defined by $p_{\mathcal{T} A}(b, v)=p_{T A}(v)=a \in A$, with $p_{T A}: T A \rightarrow A$ the canonical projection of the tangent bundle $T A$ over the base $A$. An element $(b, v)$ of $\mathcal{T} A$ will be denoted by $(a, b, v)$, where $a \in A$ is the point where $v$ is tangent to $A$. With this notation, $\mathcal{T} A=\left\{(a, b, v) \in A \times A \times T A \mid p(a)=p(b), \rho(b)=T_{a} p(v)\right.$, with $\left.v \in T_{a} A\right\}$. The vector bundle $p_{\mathcal{T} A}: \mathcal{T} A \rightarrow A$ can be endowed with a Lie algebroid structure, where the anchor is the map $\rho_{\mathcal{T} A}: \mathcal{T} A \rightarrow T A$, given by $\rho_{\mathcal{T} A}(a, b, v)=v$, and the Lie bracket on the space of sections is defined by setting $[15,18]$ :

$$
\left[V_{1}, V_{2}\right]_{\mathcal{T} A}(a)=\left(a,\left[\sigma_{1}, \sigma_{2}\right]_{A}(p(a)),\left[X_{1}, X_{2}\right](a)\right),
$$

for all $a \in A$ and all projectable sections $V_{1}, V_{2} \in \Gamma(\mathcal{T} A)$, i.e. sections of the form $V_{i}(a)=\left(a, \sigma_{i}(p(a)), X_{i}(a)\right)$ where $\sigma_{i} \in \Gamma(A)$ and $X_{i} \in \mathfrak{X}(A)$ are such that $T p \circ X_{i}=\rho\left(\sigma_{i}\right) \circ p$, with $i=1,2$. If $A$ is the tangent bundle to a manifold $Q, A=T Q$, endowed with its usual Lie algebroid structure, the prolongation of the Lie algebroid $A$ is the tangent bundle $T(T Q)$ to $T Q$ endowed with its usual structure of Lie algebroid over $T Q$ (see [18]).

Let us consider on the Lie algebroid $A$ a new set of local coordinates $\left\{\left(q^{i}, \mathbf{w}^{\alpha}\right) \mid i=1, \ldots, n, \alpha=1, \ldots, s\right\}$ (see section 4) associated to a basis of sections $\left\{f_{\alpha} \mid \alpha=1, \ldots, s\right\}$ of $A$, that satisfies:

$$
\begin{aligned}
& \mathbf{w}^{\alpha}=\widehat{\Phi_{\alpha}}(q, \mathbf{v})=\Phi_{\alpha \beta}(q) \mathbf{v}^{\beta}, \\
& \mathbf{v}^{\alpha}=\widehat{\Psi_{\alpha}}(q, \mathbf{w})=\Psi_{\alpha \beta}(q) \mathbf{w}^{\beta},
\end{aligned}
$$

for all $\alpha=1, \ldots, s$, where $\widehat{\Phi_{\alpha}}$ and $\widehat{\Phi_{\alpha}}$ are linear functions on $A$ associated to the $A$-1-forms $\Phi_{\alpha}$ and $\Psi_{\alpha}$, respectively, that verify $\Psi_{\alpha \beta} \Phi_{\beta \gamma}=\delta_{\alpha \gamma}$. With these conditions, we have the following relations:

$$
f_{\alpha}=\Psi_{\beta \alpha} e_{\beta}, \quad e_{\alpha}=\Phi_{\beta \alpha} f_{\beta}, \quad \frac{\partial}{\partial \mathbf{w}^{\alpha}}=\Psi_{\beta \alpha} \frac{\partial}{\partial \mathbf{v}^{\beta}}, \quad \frac{\partial}{\partial \mathbf{v}^{\alpha}}=\Phi_{\beta \alpha} \frac{\partial}{\partial \mathbf{w}^{\beta}} .
$$

Associated with the new coordinates on the Lie algebroid $A$, we consider on the prolongation of $A$ the following basis of local sections:

$$
\begin{aligned}
\mathcal{X}_{\alpha}^{\prime}(a) & =\left(a, f_{\alpha}(p(a)), X_{\alpha}(a)\right), \\
\mathcal{V}_{\alpha}^{\prime}(a) & =\left(a, 0,\left.\frac{\partial}{\partial \mathbf{w}^{\alpha}}\right|_{a}\right)
\end{aligned}
$$


where $X_{\alpha}=\Psi_{\beta \alpha} \rho_{\beta}^{i} \partial_{q^{i}}$, for all $\alpha=1, \ldots, r$. Thus, the structure functions of the Lie algebroid $\mathcal{T} A$ are given by:

$$
\begin{aligned}
& {\left[\mathcal{X}^{\prime}{ }_{\alpha}, \mathcal{X}^{\prime}\right]_{\mathcal{T} A}=\gamma_{a b}^{\epsilon} \mathcal{X}_{\epsilon}^{\prime}, \quad\left[\mathcal{X}^{\prime}{ }_{\alpha}, \mathcal{V}^{\prime}\right]_{\mathcal{T}_{A}}=\Psi_{\beta \alpha} \rho^{i}{ }_{\beta} \frac{\partial \Psi_{\gamma \beta}}{\partial q^{i}} \Phi_{\epsilon \gamma} \mathcal{V}^{\prime \epsilon}, \quad\left[\mathcal{V}^{\prime}{ }_{\alpha}, \mathcal{V}_{\beta}^{\prime}\right]_{\mathcal{T} A}=0} \\
& \rho_{\mathcal{T} A}\left(\mathcal{X}^{\prime}{ }_{\alpha}\right)=X_{\alpha}, \quad \rho_{\mathcal{T} A}\left(\mathcal{V}^{\prime}{ }_{\alpha}\right)=\frac{\partial}{\partial \mathbf{w}^{\alpha}}
\end{aligned}
$$

where $\left[f_{\alpha}, f_{\beta}\right]_{A}=\gamma_{a b}^{\epsilon} f_{\epsilon}$.

Let $L \in C^{\infty}(A)$ be the Lagrangian of a dynamical system on the Lie algebroid $A$, depending on the action of a non-conservative force $\mathcal{Q}$. The elements of the Lagrangian formalism on the Lie algebroid $A$, with respect to the local structure defined above, are given by:

1. Vertical endomorphism:

$$
S=\mathcal{V}_{\beta}^{\prime} \otimes \mathcal{X}^{\prime \beta}
$$

2. Liouville section:

$$
\Delta=\mathbf{w}^{\epsilon} \mathcal{V}^{\prime}{ }_{\epsilon}
$$

3. Energy (in the absence of non-conservative forces):

$$
E_{L}=\mathbf{w}^{\epsilon} \frac{\partial L}{\partial \mathbf{w}^{\epsilon}}-L
$$

4. Differential of the energy:

$$
d_{\mathcal{T} A} E_{L}=\left(\mathbf{w}^{\epsilon} X_{\beta}\left(\frac{\partial L}{\partial \mathbf{w}^{\epsilon}}\right)-X_{\beta}(L)\right) \mathcal{X}^{\prime \beta}+\mathbf{w}^{\epsilon} \frac{\partial^{2} L}{\partial \mathbf{w}^{\beta} \partial \mathbf{w}^{\epsilon}} \mathcal{V}^{\prime \beta}
$$

5. Cartan 1-form:

6. Cartan 2-form:

$$
\theta_{L}=\frac{\partial L}{\partial \mathbf{w}^{\beta}} \mathcal{X}^{\prime \beta}
$$

$\omega_{L}=\frac{1}{2}\left(\gamma_{\alpha \beta}^{\epsilon} \frac{\partial L}{\partial \mathbf{w}^{\epsilon}}-X_{\alpha}\left(\frac{\partial L}{\partial \mathbf{w}^{\beta}}\right)+X_{\beta}\left(\frac{\partial L}{\partial \mathbf{w}^{\alpha}}\right)\right) \mathcal{X}^{\prime \alpha} \wedge \mathcal{X}^{\prime \beta}+\frac{\partial^{2} L}{\partial \mathbf{w}^{\beta} \partial \mathbf{w}^{\alpha}} \mathcal{X}^{\prime \alpha} \wedge \mathcal{V}^{\prime \beta}$

Given a section $X=a^{\alpha} \mathcal{X}^{\prime}{ }_{\alpha}+b^{\alpha} \mathcal{V}^{\prime}{ }_{\alpha}$ of $\mathcal{T} A$, the contraction of the section with the Cartan 2-form is given by

$$
\begin{aligned}
i(X) \omega_{L}= & {\left[a^{\alpha}\left[\gamma_{\alpha \beta}^{\epsilon} \frac{\partial L}{\partial \mathbf{w}^{\epsilon}}-X_{\alpha}\left(\frac{\partial L}{\partial \mathbf{w}^{\beta}}\right)+X_{\beta}\left(\frac{\partial L}{\partial \mathbf{w}^{\alpha}}\right)\right]-b^{\alpha} \frac{\partial^{2} L}{\partial \mathbf{w}^{\alpha} \partial \mathbf{w}^{\beta}}\right] \mathcal{X}^{\prime \beta} } \\
& +a^{\alpha} \frac{\partial^{2} L}{\partial \mathbf{w}^{\beta} \partial \mathbf{w}^{\alpha}} \mathcal{V}^{\prime \beta} .
\end{aligned}
$$


Therefore, if the Lagrangian is regular, the dynamics has a unique solution $X=a^{\alpha} \mathcal{X}^{\prime}{ }_{\alpha}+b^{\alpha} \mathcal{V}^{\prime}{ }_{\alpha}$ that satisfies the system

$$
\left\{\begin{array}{l}
a^{\alpha}=\mathbf{w}^{\alpha} \\
b^{\alpha}=\bar{W}^{\alpha \beta}\left[\mathbf{w}^{\zeta} \gamma_{\zeta \beta}^{\epsilon} \frac{\partial L}{\partial \mathbf{w}^{\epsilon}}-\mathbf{w}^{\zeta} X_{\zeta}\left(\frac{\partial L}{\partial \mathbf{w}^{\beta}}\right)+X_{\beta}(L)+\Upsilon_{\beta}\right],
\end{array}\right.
$$

where $\bar{W}^{\alpha \beta}$ represents the entries of the inverse matrix of $\left(\partial^{2} L / \partial \mathbf{w}^{\beta} \partial \mathbf{w}^{\alpha}\right)$ and $\Upsilon_{\beta}=\Psi_{\alpha \beta} Q_{\alpha}$ is the $\beta$-component of the non-conservative force $\mathcal{Q}=Q_{\alpha} \mathcal{X}^{\alpha}$, in the new coordinates. As we expect the solution of the dynamics is a SODE section of $\mathcal{T} A$, because $S(X)=\Delta$. Since $X$ is a SODE vector field, the dynamics equation is equivalent to

$$
£_{\rho_{\mathcal{T} A}}(X) \theta_{L}=d_{\mathcal{T} A} L+\mathcal{Q},
$$

where $£_{\rho_{\mathcal{T} A}}(X):=i(X) \circ d_{\mathcal{T}_{A}}+d_{\mathcal{T}_{A}} \circ i(X)$. The generalized Euler-Lagrange equations in the new coordinates, are given by

$$
\frac{d}{d t}\left(\frac{\partial L}{\partial \mathbf{w}^{\alpha}}\right)=\Psi_{\beta \alpha} \rho^{i} \frac{\partial L}{\partial q^{i}}+\mathbf{w}^{\epsilon} \gamma_{\epsilon \alpha}^{\beta} \frac{\partial L}{\partial \mathbf{w}^{\beta}}+\Upsilon_{\alpha}
$$

where $\dot{q}^{i}=\mathbf{w}^{\alpha} \Psi_{\beta \alpha} \rho_{\beta}^{i}$.

Example 6.1. Let $P(M, G)$ be a principal bundle and $\left(q^{i}, g^{I}, \dot{q}^{i}, \xi^{I}\right)$ a set of quasi-coordinates on TP, where $\left(q^{i}\right)$ is a set of local coordinates on $M,\left(q^{i}, \dot{q}^{i}\right)$ is the usual set of local coordinates on the tangent bundle $T M$ and $\left(g^{I}, \xi^{I}\right)$ is a set of quasi-coordinates on TG, given in the example 5.2.

Given a regular and $G$-invariant Lagrangian $L \in C^{\infty}(T P)$, it does not depend on the elements of the Lie group $G$. Thus, in the quasi-coordinates, the Lagrangian is given by $l(q, \dot{q}, \xi)=L_{q c}(q, g, \dot{q}, \xi)=L(q, g, \dot{q}, \dot{g})$. Since the Lagrangian on the Lie algebroid TP is G-invariant, we can obtain the equations of motion on the gauge algebroid $T P / G$ from equations (13), in the absence of non-conservative forces:

$$
\begin{aligned}
\frac{d}{d t}\left(\frac{\partial l}{\partial \xi^{I}}\right) & =\xi^{J} \gamma_{J I}^{K} \frac{\partial l}{\partial \xi^{K}}, \\
\frac{d}{d t}\left(\frac{\partial l}{\partial \dot{q}^{i}}\right) & =\frac{\partial l}{\partial q^{i}},
\end{aligned}
$$

where $\gamma_{J I}^{K}=c_{J I}{ }^{K}$ are the structure constants of the Lie algebra $\mathfrak{g}$ of the Lie group $G$, with respect to a basis $\left\{e_{I}\right\}$ of $\mathfrak{g} \equiv T_{e} G$ (see [14]). Indeed, let $\Pi: T P \rightarrow T P / G$ be the canonical projection over the principal bundle 
$\pi: P \rightarrow M=P / G$. The gauge algebroid structure $\left(\rho_{T P / G},[\cdot, \cdot]_{T P / G}\right)$ is given by (see for example $[5,17])$ :

(1) $\rho_{T P / G}(\widehat{X})=T \pi \circ \rho_{T P}(X)$,

(2) $[\widehat{X}, \widehat{Y}]_{T P / G}=\Pi \circ[X, Y]_{T P}$,

for all $X, Y \in \Gamma^{\Pi}(T P)$, П-related with $\widehat{X}, \widehat{Y} \in \Gamma(T P / G)$, respectively. Given a connection on the principal bundle $\pi: P \rightarrow M$, we denote by $\mathcal{A}$ the connection 1-form associated with the principal connection; in local coordinates, $\mathcal{A}\left(\partial /\left.\partial q^{i}\right|_{q}\right)=A_{i}^{I}(q) e_{I}$. Let $\left\{e_{i}, e_{I}\right\}$ be a basis of local sections of $T P / G$, obtained by the $\Pi$-projection of the basis of local sections $\left\{\left(\partial / \partial q^{i}\right)^{h}, e_{I}^{L}\right\}$ of TP, where $\left(\partial / \partial q^{i}\right)^{h}=\partial_{q^{i}}-A_{i}^{I} e_{I}^{L}$ is the horizontal lift of the vector field $\partial_{q^{i}}$ on $M$ to a vector field on $P$ and $e_{I}^{L}$ is the left-invariant vector field on $G$ associated with the element $e_{I}$ of the basis of $\mathfrak{g}$. In these local coordinates, the gauge algebroid structure is given by:

$$
\begin{aligned}
& {\left[e_{i}, e_{j}\right]_{T P / G}=-C_{i j}^{K} e_{K} \quad\left[e_{i}, e_{I}\right]_{T P / G}=c_{I J}{ }^{K} A_{i}^{J} e_{K}, \quad\left[e_{I}, e_{J}\right]_{T P / G}=c_{I J}{ }^{K} e_{K},} \\
& \rho_{T P / G}\left(e_{i}\right)=\frac{\partial}{\partial q^{i}}, \quad \rho_{T P / G}\left(e_{J}\right)=0,
\end{aligned}
$$

where $C_{i j}^{K}$ are the coefficients of the curvature form defined by the principal connection. The local basis of sections $\left\{\partial_{q^{i}}, e_{I}^{L}\right\}$ of TP is associated to the set of local coordinates given initially, $\left(q^{i}, g^{I}, \dot{q}^{i}, \xi^{I}\right)$. The П-projections, $\Pi \circ \partial_{q^{i}}=f_{i} \circ \pi$ and $\Pi \circ e_{I}^{L}=f_{I} \circ \pi$, define a local base of sections $\left\{f_{i}, f_{I}\right\}$ of TP/G, associated to the local coordinates $\left(q^{i}, \dot{q}^{i}, \xi^{I}\right)$ on $T P / G$. Note that $f_{i}=e_{i}+A_{i}^{I} e_{I}$ and $f_{I}=e_{I}$. By the definition of the gauge algebroid structure, we have $\left[f_{i}, f_{j}\right]_{T P / G}=\left[f_{i}, f_{I}\right]_{T P / G}=0$ and $\left[f_{I}, f_{J}\right]_{T P / G}=c_{I J}{ }^{K} f_{K}$. So, using the reduced Lagrangian $l \in C^{\infty}(T P / G)$, we obtain from the generalized Euler-Lagrange equations (13) the equations of motion (14). The reduced dynamics $\widehat{X}_{l}=a^{\alpha} \mathcal{X}_{\alpha}^{\prime}+b^{\alpha} \mathcal{V}_{\alpha}^{\prime}$ satisfies the following system

$$
\left\{\begin{array}{l}
a^{\alpha}=\mathbf{w}^{\alpha} \\
b^{\alpha}=\bar{W}^{\alpha \beta}\left[\xi^{J} \gamma_{J \beta}^{K} \frac{\partial l}{\partial \xi^{K}}-\dot{q}^{i} \frac{\partial^{2} l}{\partial q^{i} \partial \mathbf{w}^{\beta}}+\delta_{\beta i} \frac{\partial l}{\partial q^{i}}\right],
\end{array}\right.
$$

where $\mathbf{w}^{i}=\dot{q}^{i}, \mathbf{w}^{I}=\xi^{I}, \bar{W}^{\alpha \beta}$ are the entries of the inverse matriz of $\left(\partial^{2} L / \partial \mathbf{w}^{\beta} \partial \mathbf{w}^{\alpha}\right)$ and $\left\{\mathcal{X}_{\alpha}^{\prime}, \mathcal{V}_{\alpha}^{\prime}\right\}$ is a base of local sections of $\mathcal{T}(T P / G)$ defined in (12).

From Carinena et. al. [6], the Lagrangian $L$ is П-projectable because there exists a function $l$ such that $L=l \circ \Pi$. Therefore, the solution $X_{L} \in$ 
$\mathfrak{X}(T P)$ of the dynamics characterized by L, projects into the dynamics $\widehat{X}_{l} \in$ $\Gamma(\mathcal{T}(T P / G))$ characterized by the reduced lagrangian $l$, that is, $\bar{\Pi} \circ X_{L}=$ $\widehat{X}_{l} \circ \Pi$, where $\bar{\Pi}: \mathcal{T}(T P) \equiv T(T P) \rightarrow \mathcal{T}(T P / G) \equiv T(T P) / G$ is defined by $\bar{\Pi}(a, b, v)=(\Pi(a), \Pi(b), T \Pi(v))$, for all $(a, b, v) \in \mathcal{T}(T P)$.

\section{Application of the above problem to solve Lagrangian systems with non-holonomic linear constraints on a Lie algebroid}

In this section, we intend to solve systems with linear non-holonomic constraints on a Lie algebroid $A$, i.e. constraints which are linear in the local coordinates $\mathbf{v}^{\alpha}$ on $A$ associated with a local base of sections $\left\{e_{\alpha}\right\}$ of $A$, by using local coordinates adapted to the constraints.

Let us consider on a Lie algebroid $\left(A, \rho,[\cdot, \cdot]_{A}\right)$ over $M$, a system with $k$ linear non-holonomic constraints

$$
\phi_{a}(q, \mathbf{v})=\widehat{\Phi_{a}}(q, \mathbf{v})=\Phi_{a \beta}(q) \mathbf{v}^{\beta},
$$

given by a subbundle $\tau: B \rightarrow M$ of $A$, where $\Phi_{a}$ is an $A$-1-form and $\widehat{\Phi_{a}}$ is the associated linear function. The submanifold $B$ is defined by the set $\left\{\phi_{a}=0 \mid a=1, \ldots, k\right\}$ and is called the constrained manifold. Suppose that the $A$-1-forms $\Phi_{a}$ are such that $\Phi_{1} \wedge \ldots \wedge \Phi_{k} \neq 0$. Then, the functions $\phi_{a}$ are functionally independent.

Let $\mathcal{T} B$ be the vector bundle over $B$, whose total space

$$
\mathcal{T} B=\left\{(b, c, v) \in B \times B \times T B \mid \tau(b)=\tau(c), \varrho(c)=T \tau(v) \operatorname{com} v \in T_{b} B\right\}
$$

is given by the pullback of the vector bundle $T \tau: T B \rightarrow T M$ by the map $\varrho=\rho \circ \iota: B \rightarrow T M$, where $\iota: B \rightarrow A$ is the canonical inclusion. The projection $p_{\mathcal{T} B}: \mathcal{T} B \rightarrow B$ of $\mathcal{T} B$ onto $B$, is given by $p_{\mathcal{T} B}(b, c, v)=b$. Let us suppose that $L \in C^{\infty}(A)$ is a regular Lagrangian, that describes the nonholonomic system subjected to the action of a non-conservative force $\mathcal{Q}$. In parallel with the formalism of linear non-holonomic systems in a tangente bundle, the equations of motion of the non-holonomic system in $A$, can be written satisfying the d'Alembert-Chetaev principle, in the global form:

$$
\left\{\begin{array}{l}
\left.\left(i(X) \omega_{L}-d_{\mathcal{T} A} E_{L}+\mathcal{Q}\right)\right|_{B} \in \Gamma\left(\widetilde{B^{0}}\right) \\
\left.X\right|_{B} \in \Gamma(\mathcal{T} B)
\end{array},\right.
$$

where $B^{0}=\left\langle\Phi_{a} \mid a=1, \ldots, k\right\rangle$ is the annihilator of $B$, and $\widetilde{B^{0}}=$ $\left\langle p_{2}^{*} \Phi_{a} \mid a=1, \ldots, k\right\rangle$ can be seen as a vector bundle over $B$, where $p_{2}: \mathcal{T} A \rightarrow$ 
$A$ is the projection defined by $p_{2}(a, b, v)=b$, for all $(a, b, v) \in \mathcal{T} A$. Note that, for each $m \in M, B_{m}^{0}$ are the set generated by the elements $\Phi_{a}(m)$ that satisfy $\left\langle\Phi_{a}(m), v\right\rangle=0$, for each element $v \in B_{m}$ and $a=1, \ldots, k$.

Suppose that the admissibility condition holds:

$$
\operatorname{dim}\left(\mathcal{T}_{b}^{A} B\right)^{0}=\operatorname{dim} S^{*}\left(\left(\mathcal{T}_{b}^{A} B\right)^{0}\right),
$$

for all $b \in B$, where $\mathcal{T}^{A} B \rightarrow B$ is the prolongation of the subbundle $B$ with respect to the Lie algebroid $A$ (see $[15,19]$ ) and $S$ is the vertical endomorphism on $\mathcal{T} A$. Observe that $\operatorname{dim}\left(\mathcal{T}^{A} B\right)^{0}=k$ and $\left(\mathcal{T}^{A} B\right)^{0}$ is generated by the 1-forms $d_{\mathcal{T} A} \phi_{a}$, and also $S^{*}\left(\left(\mathcal{T}^{A} B\right)^{0}\right)=\widetilde{B^{0}}$.

7.1. Lagrange multipliers method in a Lie algebroid framework. In this section, we will solve the non-holonomic system given previous, using the method of Lagrangian multipliers (see [4] for the classical case).

The dynamics equation of the system is given by

$$
i(X) \omega_{L}=d_{\mathcal{T} A} E_{L}-\mathcal{Q}-\lambda_{a} p_{2}^{*} \Phi_{a}
$$

where the Lagrange multipliers $\lambda_{a} \in C^{\infty}(A)$ are to be determined by the tangency condition $£_{\rho_{\mathcal{T} A}}(X) \phi_{a}=0$, for all $a=1, \ldots, k$. Recall that $p_{2}: \mathcal{T} A \rightarrow$ $A$ is defined by $p_{2}(a, b, v)=b$ and the semi-basic sections $p_{2}^{*} \Phi_{a}=\Phi_{a \beta} \chi^{\beta}$ are the reaction forces of the Lie algebroid $A$ (see [6]). The solution of the equation (16) is a section of the form

$$
X=X_{L}^{\mathcal{Q}}+\lambda_{a} Z_{a}
$$

where $X_{L}^{\mathcal{Q}}$ is the solution of the dynamics without constraints and $Z_{a}$ is a vertical section of $\mathcal{T} A$ satisfying the condition

$$
i\left(Z_{a}\right) \omega_{L}=-p_{2}^{*} \Phi_{a} .
$$

Note, once again, that the section $X$ is a SODE section of $\mathcal{T} A$, since $S(X)=$ $S\left(X_{L}^{\mathcal{Q}}\right)=\Delta$.

It is important to observe that for the Lagrangian multipliers $\lambda_{a}$ to be determined, we must suppose the following compatibility condition (see [8]): the matriz of entries $\mathcal{C}_{a b}=\rho_{\mathcal{T} A}\left(Z_{a}\right) \phi_{b}$ is regular in each point of $B$, where $B=$ $\left\{\mathbf{w}^{\alpha}=0 \mid \alpha=s-k+1, \ldots, s\right\}$ is the constrained manifold. In this situation, we say that the non-holonomic system $(L, B)$ on the Lie algebroid $A$ is regular. So, let us suppose that the non-holonomic system $(L, B)$ is regular. 
We can assume without losing generality that the last $k$ columns of the matrix $\left(\Phi_{a \beta}\right)$ are independent. Thus, let us consider on the bundle $A$ a set of coordinates $\left(q^{1}, \ldots, q^{n}, \mathbf{w}^{1}, \ldots, \mathbf{w}^{s}\right)$ adapted to the constraints:

$$
\begin{aligned}
\mathbf{w}^{\alpha} & =\mathbf{v}^{\alpha}, \quad \forall \alpha=1, \ldots,(s-k), \\
\mathbf{w}^{s-k+a} & =\phi_{a}, \quad \forall a=1, \ldots, k .
\end{aligned}
$$

The coordinates transformation matrices are given by:

$$
\widetilde{\Phi}=\left(\begin{array}{cc}
I_{s-k} & 0_{(s-k) \times k} \\
A_{21} & A_{22}
\end{array}\right), \quad \widetilde{\Psi}=\left(\begin{array}{cc}
I_{s-k} & 0_{(s-k) \times k} \\
B_{21} & B_{22}
\end{array}\right),
$$

where the matrix $A=\left(A_{21} A_{22}\right)$ is given by $A_{a b}=\Phi_{a b}$, for all $a=1, \ldots, k$ and $b=1, \ldots, s$, with $A_{22}$ invertible by hypothesis, and the matrix $B=\left(B_{21} B_{22}\right)$ is given by $B_{21}=-A_{22}^{-1} A_{21}$ e $B_{22}=A_{22}^{-1}$. Note that the matrices satisfy $\widetilde{\Phi} \widetilde{\Psi}=I_{s}=\widetilde{\Psi} \widetilde{\Phi}$. In the new coordinates the geometrical dynamics solution is given by

$$
\left.X\right|_{B}=\mathbf{w}^{\underline{\alpha}} \mathcal{X}_{\underline{\alpha}}^{\prime}+f^{\underline{\alpha}}(q, \mathbf{w}) \mathcal{V}_{\underline{\alpha}}^{\prime},
$$

where $\underline{\alpha}=1, \ldots, s-k$. The functions $f \underline{\alpha}$ are determined by

$$
f^{\underline{\alpha}}(q, \mathbf{w})=g^{\underline{\alpha}}(q, \mathbf{w})+\lambda_{a} \mathcal{W}^{\underline{\alpha} \beta} \Phi_{a \beta},
$$

with $\beta=1, \ldots, s$ and $a=1, \ldots, k$, where

$$
\begin{aligned}
X_{L}^{\mathcal{Q}} & =\mathbf{w}^{\beta} \mathcal{X}_{\beta}^{\prime}+g^{\beta}(q, \mathbf{w}) \mathcal{V}_{\beta}^{\prime}, \\
Z_{a} & =\mathcal{W}^{\alpha \beta} \Phi_{a \beta} \mathcal{V}_{\alpha}^{\prime},
\end{aligned}
$$

and the function $\lambda_{a}$ is given by

$$
d_{\mathcal{T} A} \mathbf{w}^{n-k+b}\left(X_{L}^{\mathcal{Q}}\right)+\lambda_{a} d_{\mathcal{T} A} \mathbf{w}^{n-k+b}\left(Z_{a}\right)=0, \quad \forall b=1, \ldots, k .
$$

Therefore, the dynamics is given by the integral curves of the following vector field in $B$

$$
\rho_{\mathcal{T} A}\left(\left.X\right|_{B}\right)=\mathbf{w}^{\underline{\alpha}} X_{\underline{\alpha}}+f^{\underline{\alpha}} \frac{\partial}{\partial \mathbf{w}^{\underline{\alpha}}} .
$$

Since the solution $X$ is a $\mathrm{SODE}$ section of $\mathcal{T} A$, it satisfies the following equation

$$
£_{\rho_{\mathcal{T} A}}(X) \theta_{L}=d_{\mathcal{T} A} L+\mathcal{Q}+\lambda_{a} p_{2}^{*} \Phi_{a} .
$$

In the new coordinates, the previous equation is given on $B$ by

$$
\frac{d}{d t}\left(\frac{\partial L}{\partial \mathbf{w}^{\alpha}}\right)=\widetilde{\Psi}_{\beta \alpha} \rho^{i}{ }_{\beta} \frac{\partial L}{\partial q^{i}}+\mathbf{w}^{\underline{\epsilon}} \gamma_{\underline{\epsilon} \alpha}^{\beta} \frac{\partial L}{\partial \mathbf{w}^{\beta}}+\Upsilon_{\alpha}+\lambda_{a} \widetilde{\Psi}_{\beta \alpha} \Phi_{a \beta},
$$


where $\dot{q}^{i}=\mathbf{w}^{\underline{\alpha}} \widetilde{\Psi}_{\beta \underline{\alpha}} \rho_{\beta}^{i}$ and $\Upsilon_{\alpha}=\widetilde{\Psi}_{\beta \alpha} Q_{\beta}$ is the $\alpha$-component of the nonconservative force $\mathcal{Q}$ on the new coordinates, with $\underline{\alpha}, \underline{\epsilon}=1, \ldots, s-k, \alpha, \beta=$ $1, \ldots, s, a=1, \ldots, k$ e $i=1, \ldots, n$. Thus, we have

$$
\left\{\begin{array}{l}
\dot{q}^{i}=\mathbf{w}^{\underline{\alpha}} \widetilde{\Psi}_{\beta \underline{\alpha}} \rho^{i}{ }_{\beta} \\
\frac{d}{d t}\left(\frac{\partial L}{\partial \mathbf{w}^{\underline{\alpha}}}\right)=\widetilde{\Psi}_{\beta \underline{\alpha}} \rho_{\beta}^{i} \frac{\partial L}{\partial q^{i}}+\mathbf{w}^{\underline{\epsilon}} \gamma_{\underline{\underline{\epsilon}} \underline{\alpha}}^{\beta} \frac{\partial L}{\partial \mathbf{w}^{\beta}}+\Upsilon_{\underline{\alpha}} \\
\frac{d}{d t}\left(\frac{\partial L}{\partial \mathbf{w}^{\bar{\alpha}}}\right)=\widetilde{\Psi}_{\beta \bar{\alpha}} \rho_{\beta}^{i} \frac{\partial L}{\partial q^{i}}+\mathbf{w}^{\underline{\epsilon}} \gamma_{\underline{\underline{\epsilon}} \bar{\alpha}}^{\beta} \frac{\partial L}{\partial \mathbf{w}^{\beta}}+\Upsilon_{\bar{\alpha}}+\lambda_{\bar{\alpha}-s+k}
\end{array},\right.
$$

with $\bar{\alpha}=s-k+1, \ldots, s$. Eliminating the Lagrange multipliers from the system we obtain, in the new coordinates, the following generalized Lagranged'Alembert equation

$$
\frac{d}{d t}\left(\frac{\partial L}{\partial \mathbf{w}^{\underline{\alpha}}}\right)=\widetilde{\Psi}_{\beta \underline{\alpha}} \rho^{i}{ }_{\beta} \frac{\partial L}{\partial q^{i}}+\mathbf{w}^{\underline{\epsilon}} \gamma_{\underline{\underline{\epsilon}}}^{\beta} \frac{\partial L}{\partial \mathbf{w}^{\beta}}+\Upsilon_{\underline{\alpha}},
$$

where $\dot{q}^{i}=\mathbf{w}^{\underline{\alpha}} \widetilde{\Psi}_{\beta \underline{\alpha}} \rho_{\beta}^{i}$.

Note that we would obtain similar results if we had selected a different set of coordinates adapted to the constraints: $\mathbf{w}^{I}=\widetilde{\Phi}_{I \beta} \mathbf{v}^{\beta}$ and $\mathbf{w}^{s-k+a}=\phi_{a}=$ $\Phi_{a \beta} \mathbf{V}^{\beta}$, for all $I=1, \ldots, s-k$ and $a=1, \ldots, k$, with the only assumption that the following matrix be invertible

$$
\widetilde{\Phi}=\left(\begin{array}{c}
A \\
B
\end{array}\right)
$$

where $A_{I \beta}=\widetilde{\Phi}_{I \beta}$ and $B_{a \beta}=\Phi_{a \beta}$, for all $I=1, \ldots, s-k, a=1, \ldots, k$ and $\beta=1, \ldots, s$.

Example 7.1. Let us consider the motion of a free particle, of unity mass, in the configuration space $M=\mathbb{R}^{3}$, with a linear constraint

$$
\phi=\dot{z}-y \dot{x}
$$

In order to determined the solution of this problem, we consider on the Lie algebroid $A=T \mathbb{R}^{3}$ the set of local coordinates $\left(x, y, z, \mathbf{w}^{1}, \mathbf{w}^{2}, \mathbf{w}^{3}\right)$, given by:

$$
\mathbf{w}^{1}=v_{x}=\dot{x}, \quad \mathbf{w}^{2}=v_{y}=\dot{y}, \quad \mathbf{w}^{3}=\phi=v_{z}-y v_{x} .
$$


From the previous relations, we conclude that the coordinates transformation matrices $\widetilde{\Phi}$ and $\widetilde{\Psi}$ are:

$$
\widetilde{\Phi}=\left(\begin{array}{ccc}
1 & 0 & 0 \\
0 & 1 & 0 \\
-y & 0 & 1
\end{array}\right), \quad \widetilde{\Psi}=\left(\begin{array}{lll}
1 & 0 & 0 \\
0 & 1 & 0 \\
y & 0 & 1
\end{array}\right) .
$$

The motion of the free particle is characterized by the regular Lagrangian

$$
L=\frac{1}{2}\left(\left(\mathbf{w}^{1}\right)^{2}+\left(\mathbf{w}^{2}\right)^{2}+\left(\mathbf{w}^{3}+y \mathbf{w}^{1}\right)^{2}\right) .
$$

Let $B$ be the constraint manifold. Solving the problem through the method of Lagrangian multipliers, we obtain the geometrical solution

$$
\left.X\right|_{B}=\mathbf{w}^{1} \mathcal{X}_{1}^{\prime}+\mathbf{w}^{2} \mathcal{X}_{2}^{\prime}-\frac{\mathbf{w}^{1} \mathbf{w}^{2}}{y^{2}+1} y \mathcal{V}_{1}^{\prime},
$$

where:

$$
\begin{aligned}
X_{L} & =\mathbf{w}^{1} \mathcal{X}_{1}^{\prime}+\mathbf{w}^{2} \mathcal{X}_{2}^{\prime}+\mathbf{w}^{3} \mathcal{X}_{3}^{\prime}-\mathbf{w}^{1} \mathbf{w}^{2} \mathcal{V}_{3}^{\prime} \\
Z & =-y \mathcal{V}_{1}^{\prime}+\left(y^{2}+1\right) \mathcal{V}_{3}^{\prime} \\
\lambda & =\frac{\mathbf{w}^{1} \mathbf{w}^{2}}{y^{2}+1}
\end{aligned}
$$

In this particular case, we can identify $\mathcal{T} A=\mathcal{T}\left(T \mathbb{R}^{3}\right)$ with $T\left(T \mathbb{R}^{3}\right)=T A$. In a parallel way, we can identify the solution $\left.X\right|_{B}$ with a vector field on $B$,

$$
\left.X\right|_{B} \equiv \mathbf{w}^{1} \frac{\partial}{\partial x}+\mathbf{w}^{2} \frac{\partial}{\partial y}+y \mathbf{w}^{1} \frac{\partial}{\partial z}-\frac{\mathbf{w}^{1} \mathbf{w}^{2}}{y^{2}+1} y \frac{\partial}{\partial \mathbf{w}^{1}} .
$$

The dynamics is given by the integrable curves of the above vector field.

7.2. Gibbs-Appell's method in a Lie algebroid framework. In the formalism of Lie algebroids, the aim of Gibbs-Appell's method is to determined the equations of motion of a system with constraints. This method consist, in a first step, to determine the Gibbs-Appell's function associated with the Lagrangian of the system without constraints. After that, we need to obtain this function in a set of coordinates adapted to the constraints and, in the last step, we need to determine the equations of motion, given by the Gibbs-Appell in the new coordinates.

Next we will determine the Gibbs-Appell's function associated to a Lagrangiano $L \in C^{\infty}(A)$, defined on a Lie algebroid $\left(A, \rho,[\cdot, \cdot]_{A}\right)$ over $M$. We will show that this function is defined on a subset $A^{(2)}$ of $T A$, given by the 
set of equivalent classes of admissible curves in the bundle $p: A \rightarrow M$, i.e. curves $\sigma^{\prime}: I \rightarrow A$ in $A$ such that $\dot{\sigma}(t)=\rho\left(\sigma^{\prime}(t)\right)$, where $\sigma: I \rightarrow M$ is a curve in $M$ given by $\sigma(t)=p \circ \sigma^{\prime}(t)$. The equivalence relation between two curves of $A, \sigma_{A} \equiv\left(\sigma, \sigma^{\prime}\right)$ and $\gamma_{A} \equiv\left(\gamma, \gamma^{\prime}\right)$, is given by: the curves $\sigma_{A}$ and $\gamma_{A}$ are said to be equivalent if and only if

$$
\left\{\begin{array}{c}
\sigma(0)=\gamma(0) \\
\sigma^{\prime}(0)=\gamma^{\prime}(0) \\
\dot{\sigma}^{\prime}(0)=\dot{\gamma}^{\prime}(0)
\end{array} .\right.
$$

The set $A^{(2)}$ is an affine subbundle of $p_{T A}: T A \rightarrow A$, whose projection in $A$ is given by

$$
p_{2,1}:(q, \mathbf{v}, \mathbf{a}) \in A^{(2)} \rightarrow(q, \mathbf{v}) \in A,
$$

and the inclusion of $A^{(2)}$ in $T A$ is defined by

$$
i:\left(q^{i}, \mathbf{v}^{\alpha}, \mathbf{a}^{\alpha}\right) \in A^{(2)} \rightarrow\left(q^{i}, \mathbf{v}^{\alpha}, \mathbf{v}^{\alpha} \rho_{\alpha}^{i}, \mathbf{a}^{\alpha}\right) \in T A .
$$

So, given an element $v \in A_{a}^{(2)}$ of the form $v=\left(q^{i}, \mathbf{v}^{\alpha}, \mathbf{a}^{\alpha}\right)$, we have $i(v)=$ $\mathbf{v}^{\alpha} \rho_{\alpha}^{i} \partial / \partial q^{i}+\mathbf{a}^{\alpha} \partial / \partial \mathbf{v}^{\alpha}$.

Let $L \in C^{\infty}(A)$ be a regular Lagrangian of a system without constraints, depending on a non-conservative force $\mathcal{Q}$. To define the Gibbs-Appell's function associated to the Lagrangian $L$, we need first to consider the section of $\mathcal{T} A$ over the map $p_{2,1}: A^{(2)} \rightarrow A$

$$
\Gamma_{L}=X_{L} \circ p_{2,1}-\mathbf{T}^{(1)}
$$

where $X_{L}$ is the solution of the equation $i\left(X_{L}\right) \omega_{L}=d_{\mathcal{T} A} E_{L}$, given in local coordinates by $X_{L}(q, \mathbf{v})=\mathbf{v}^{\alpha} \mathcal{X}_{\alpha}+F^{\alpha}(q, \mathbf{v}) \mathcal{V}_{\alpha}$, and $\mathbf{T}^{(1)}$ is a section of $\mathcal{T} A$ over the map $p_{2,1}: A^{(2)} \rightarrow A$ defined by $v^{(1)}=\rho_{\mathcal{T} A} \circ T^{(1)}$, where $v^{(1)}$ is a vector field on $A$ over the map $p_{2,1}: A^{(2)} \rightarrow A$, given by

$$
v^{(1)} \circ \sigma^{2}=T \sigma^{1}(d / d t),
$$

for all admissible curves $\sigma^{1} \equiv\left(\sigma, \sigma^{\prime}\right): I \rightarrow A$, where $\sigma^{2}=\left(\sigma, \sigma^{\prime}, \dot{\sigma}^{\prime}\right): I \rightarrow$ $A^{(2)}$.

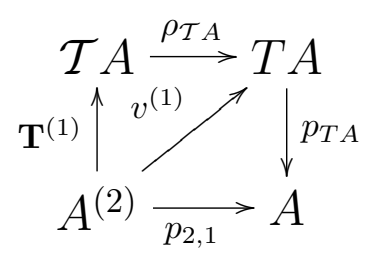


In local coordinates, the curve $\sigma^{1}$ is given by $\left(\sigma^{i}, \mathbf{v}^{\alpha}\right)$ and, since it is admissible, we have that $\dot{\sigma}^{i}=\mathbf{v}^{\alpha} \rho_{\alpha}^{i}$. Thus,

$$
v^{(1)}\left(q^{i}, \mathbf{v}^{\alpha}, \dot{\mathbf{v}}^{\alpha}\right)=\mathbf{v}^{\alpha} \rho_{\alpha}^{i}(q) \partial_{q^{i}}+\dot{\mathbf{v}}^{\alpha} \partial_{\mathbf{v}^{\alpha}} .
$$

The section $\mathbf{T}^{(1)}$ of $\mathcal{T} A$ over the map $p_{2,1}: A^{(2)} \rightarrow A$, is given in local coordinates by

$$
\mathbf{T}^{(1)}\left(q^{i}, \mathbf{v}^{\alpha}, \dot{\mathbf{v}}^{\alpha}\right)=\mathbf{v}^{\alpha} \mathcal{X}_{\alpha}\left(q^{i}, \mathbf{v}^{\alpha}\right)+\dot{\mathbf{v}}^{\alpha} \mathcal{V}_{\alpha}\left(q^{i}, \mathbf{v}^{\alpha}\right) .
$$

Therefore, the section $\Gamma_{L}$ takes values in the vertical subbundle of $\mathcal{T} A$, i.e. $p_{2} \circ \Gamma_{L}=s_{0} \circ p_{2,1}$, where $s_{0}$ is the null section of $A$; in local coordinates,

$$
\Gamma_{L}\left(q^{i}, \mathbf{v}^{\alpha}, \dot{\mathbf{v}}^{\alpha}\right)=\left(F^{\alpha}\left(q^{i}, \mathbf{v}^{\alpha}\right)-\dot{\mathbf{v}}^{\alpha}\right) \mathcal{V}_{\alpha}
$$

Let us also consider a symmetric tensor $\mathcal{G}_{A}: \mathcal{T} A \times_{A} \mathcal{T} A \rightarrow \mathbb{R}$ in $A$, given in local coordinates by

$$
\mathcal{G}_{A}(q, \mathbf{v})=\mathcal{G}_{\alpha \beta}^{1}(q, \mathbf{v}) \mathcal{V}^{\alpha} \otimes \mathcal{V}^{\beta}+\mathcal{G}_{\alpha \beta}^{2}(q, \mathbf{v}) \mathcal{V}^{\alpha} \otimes \mathcal{X}^{\beta}+\mathcal{G}_{\alpha \beta}^{3}(q, \mathbf{v}) \mathcal{X}^{\alpha} \otimes \mathcal{X}^{\beta},
$$

such that $S^{*} \mathcal{G}_{A}=p_{2}^{*} \mathcal{G}$, where $\mathcal{G}: A \times_{M} A \rightarrow \mathbb{R}$ is the fundamental tensor associated to the Lagrangian function $L \in C^{\infty}(A)$, given in local coordinates by $\mathcal{G}(q)=\mathcal{G}_{\alpha \beta}(q) e^{\alpha} \otimes e^{\beta}$, where $\mathcal{G}_{\alpha \beta}=\partial^{2} L / \partial \mathbf{v}^{\alpha} \partial \mathbf{v}^{\beta}$. In this way, $\mathcal{G}_{\alpha \beta}^{1}(a)=$ $\mathcal{G}_{\alpha \beta}(p(a))$, for all $a \in A$. Thus, the Gibbs-Appell's function associated to the Lagrangian $L$ is a function on $A^{(2)}$, defined by

$$
G_{L}=\frac{1}{2} \widetilde{\mathcal{G}}\left(\Gamma_{L}, \Gamma_{L}\right)
$$

where $\widetilde{\mathcal{G}}=\mathcal{G}_{A} \circ p_{2,1}$.

Let us consider a dynamical system on the Lie algebroid $A$ with $k$ linear non-holonomic constraints $\phi_{a}(q, \mathbf{v})=\widehat{\Phi_{a}}(q, \mathbf{v})=\Phi_{a \beta}(q) \mathbf{v}^{\beta}$, and let $\left(q^{1}, \ldots, q^{n}, \mathbf{w}^{1}, \ldots, \mathbf{w}^{s}\right)$ be a set of coordinates in $A$ adapted to the constraints:

$$
\mathbf{w}^{\alpha}=\widetilde{\Phi}_{\alpha \beta} \mathbf{v}^{\beta}
$$

where the last $k$ coordinates coincide with the constraints $\phi_{a}$, that is,

$$
\begin{aligned}
\mathbf{w}^{I} & =\widetilde{\Phi}_{I \beta} \mathbf{v}^{\beta}, \quad \forall I=1, \ldots,(s-k), \\
\mathbf{w}^{s-k+a} & =\phi_{a}, \quad \forall a=1, \ldots, k .
\end{aligned}
$$

The coordinate transformation matrix $\widetilde{\Phi}$ is invertible, so $\mathbf{v}^{\alpha}=\widetilde{\Psi}_{\alpha \beta} \mathbf{w}^{\beta}$, where $\widetilde{\Phi}_{\gamma \alpha} \widetilde{\Psi}_{\alpha \beta}=\delta_{\gamma \beta}$. The Gibbs-Appell function $G_{L}$, defined in a curve $\left(q^{i}, \mathbf{v}^{\alpha}, \dot{\mathbf{v}}^{\alpha}\right)$ 
in $A^{(2)}$, is given by

$G_{L}(q, \mathbf{v}, \dot{\mathbf{v}})=\frac{1}{2} \mathcal{G}_{\alpha \beta}(q) \dot{\mathbf{v}}^{\alpha} \dot{\mathbf{v}}^{\beta}-\mathcal{G}_{\alpha \beta}(q) \dot{\mathbf{v}}^{\alpha} F^{\beta}(q, \mathbf{v})+\frac{1}{2} \mathcal{G}_{\alpha \beta}(q) F^{\alpha}(q, \mathbf{v}) F^{\beta}(q, \mathbf{v})$.

In the set where the constraints $\mathbf{w}^{s-k+a}=\phi_{a}$ and their time derivatives are equal to zero, the function $G_{L}$ is easily written in the new coordinates $\left(q^{1}, \ldots, q^{n}, \mathbf{w}^{1}, \ldots, \mathbf{w}^{s-k}, \dot{\mathbf{w}}^{1}, \ldots, \dot{\mathbf{w}}^{s-k}\right)$. Note that the time derivative of a function $f \in C^{\infty}(A)$ is a function in $C^{\infty}\left(A^{(2)}\right)$, given by

$$
d_{\mathbf{T}^{(1)}} f=i\left(\mathbf{T}^{(1)}\right) d_{\mathcal{T} A} f=v^{(1)} f,
$$

where $\mathbf{T}^{(1)}$ is a section of $\mathcal{T} A$ over the map $p_{2,1}: A^{(2)} \rightarrow A$, previously defined.

To determine the equations of motion of the non-holonomic system depending on a non-conservative force $\mathcal{Q}$, we need to solve the system given by the Gibbs-Appell $(s-k)$-equations

$$
\frac{\partial G_{L}(q, \mathbf{w}, \dot{\mathbf{w}})}{\partial \dot{\mathbf{w}}^{I}}=\Upsilon_{I}
$$

where $\Upsilon_{I}=\widetilde{\Psi}_{\beta I} Q_{\beta}$ is the $I$-component of the non-conservative force, in the new coordinates, with $I=1, \ldots, s-k$. Let $B$ be the constrained manifold. The geometrical solution of the non-holonomic system is the following section of $\mathcal{T} B$

$$
X=\mathbf{w}^{I} \mathcal{X}_{I}^{\prime}+\dot{\mathbf{w}}^{I} \mathcal{V}_{I}^{\prime}
$$

where $\left\{\mathcal{X}_{\alpha}^{\prime}, \mathcal{V}_{\alpha}^{\prime}\right\}$ is given by (12), that is,

$$
X(a)=\left(a, \mathbf{w}^{I} f_{I}(m), \mathbf{w}^{I} X_{I}(a)+\left.\dot{\mathbf{w}^{I}} \frac{\partial}{\partial \mathbf{w}^{I}}\right|_{a}\right),
$$

for all $a \in A_{m}$, where $X_{I}=\widetilde{\Psi}_{\beta I} \rho^{i}{ }_{\beta} \partial_{q^{i}}$. Therefore, the dynamics is given by the integral curves of the following vector field on $B$

$$
\rho_{\mathcal{T} A}(X)=\mathbf{w}^{I} X_{I}+\dot{\mathbf{w}}^{I} \frac{\partial}{\partial \mathbf{w}^{I}} .
$$

Example 7.2. Let $A=T \mathbb{R}^{2} \times \mathbb{R}^{3} \rightarrow \mathbb{R}^{2}$ be a vector bundle, whose local coordinates $\left(x, y, \dot{x}, \dot{y}, \omega_{x}, \omega_{y}, \omega_{z}\right)$ are associated the the local basis of sections $\left\{e_{1}=\left(\partial_{x}, 0\right), e_{2}=\left(\partial_{y}, 0\right), e_{3}=\left(0, X_{3}\right), e_{4}=\left(0, X_{4}\right), e_{5}=\left(0, X_{5}\right)\right\}$ of $A$. The vector bundle $A$ is endowed with a Lie algebroid structure $\left(\rho,[\cdot, \cdot]_{A}\right)$, given locally by:

$\left[e_{3}, e_{4}\right]_{A}=-e_{5}, \quad\left[e_{3}, e_{5}\right]_{A}=e_{4}, \quad\left[e_{4}, e_{5}\right]_{A}=-e_{3}, \quad \rho\left(e_{1}\right)=\partial_{x}, \quad \rho\left(e_{2}\right)=\partial_{y}$, 
where the remaining structure functions are zero. Let us suppose that a nonholonomic system on the Lie algebroid $A$ is characterized by the regular Lagrangian

$$
L=\frac{1}{2}\left(\dot{x}^{2}+\dot{y}^{2}\right)+\frac{k^{2}}{2}\left(\omega_{x}^{2}+\omega_{y}^{2}+\omega_{z}^{2}\right),
$$

whose constraints are given by:

$$
\begin{aligned}
& \phi_{1}=\dot{x}-r \omega_{y}, \\
& \phi_{2}=\dot{y}+r \omega_{x},
\end{aligned}
$$

where $k, r$ are constants (see [8]). The solution of the system without constraints is a section of $\mathcal{T} A$, that is written in local coordinates as

$$
X_{L}=\dot{x} \mathcal{X}_{1}+\dot{y} \mathcal{X}_{2}+\omega_{x} \mathcal{X}_{3}+\omega_{y} \mathcal{X}_{4}+\omega_{z} \mathcal{X}_{5}
$$

where $\mathcal{X}_{1}(a)=\left(a, e_{1}(m),\left.\partial_{x}\right|_{a}\right), \mathcal{X}_{2}(a)=\left(a, e_{2}(m),\left.\partial_{y}\right|_{a}\right)$ and $\mathcal{X}_{i}(a)=$ $\left(a, e_{i}(m), 0\right)$, for all $a \in A_{m}$ and $i=3,4,5$. Thus, the Gibbs-Appell's function associated with $L$ is given for

$$
G_{L}=\frac{1}{2}\left[\left(\dot{\mathbf{v}}^{1}\right)^{2}+\left(\dot{\mathbf{v}}^{2}\right)^{2}\right]+\frac{k^{2}}{2}\left[\left(\dot{\mathbf{v}}^{3}\right)^{2}+\left(\dot{\mathbf{v}}^{4}\right)^{2}+\left(\dot{\mathbf{v}}^{5}\right)^{2}\right] .
$$

Let us consider the following coordinates:

$$
\begin{aligned}
& \mathbf{w}^{1}=\mathbf{v}^{1}=\dot{x}, \mathbf{w}^{2}=\mathbf{v}^{2}=\dot{y}, \mathbf{w}^{3}=\mathbf{v}^{5}=\omega_{z}, \\
& \mathbf{w}^{4}=\phi_{1}=\mathbf{v}^{1}-r \mathbf{v}^{4}, \mathbf{w}^{5}=\phi_{2}=\mathbf{v}^{2}+r \mathbf{v}^{3}
\end{aligned}
$$

Taking the constraints and their time derivatives equal to zero, we obtain

$$
G_{L}=\frac{1}{2}\left[\left(\dot{\mathbf{w}}^{1}\right)^{2}+\left(\dot{\mathbf{w}}^{2}\right)^{2}\right]+\frac{k^{2}}{2}\left[\left(\frac{-\dot{\mathbf{w}}^{2}}{r}\right)^{2}+\left(\frac{\dot{\mathbf{w}}^{1}}{r}\right)^{2}+\left(\dot{\mathbf{w}}^{3}\right)^{2}\right] .
$$

Solving the Gibbs-Appell equations, we conclude that

$$
\dot{\mathbf{w}}^{1}=\dot{\mathbf{w}}^{2}=\dot{\mathbf{w}}^{3}=0 .
$$

Therefore, the geometrical solution of the non-holonomic is given by

$$
X=\mathbf{w}^{1} \mathcal{X}_{1}^{\prime}+\mathbf{w}^{2} \mathcal{X}_{2}^{\prime}+\mathbf{w}^{3} \mathcal{X}_{3}^{\prime},
$$

that is,

$$
X(a)=\left(a, \dot{x} f_{1}(m)+\dot{y} f_{2}(m)+\omega_{z} f_{3}(m),\left.\dot{x} \partial_{x}\right|_{a}+\left.\dot{y} \partial_{y}\right|_{a}\right),
$$

for all $a \in A_{m}$. 


\section{Conclusions}

The Lie algebroid formalism is an important geometrical tool to study classical mechanics, describing it as well as in usual coordinates as in quasicoordinates. We believe that the geometrical description we presented makes the resolution of systems using quasi-coordinates in a tangent bundle easier.

We can use the Lie algebroid formalism to study systems with linear non-holonomic constraints, in parallel with the study of this type of systems in classical mechanics on a tangent bundle. Again, the rôle of "quasicoordinates" is essential to solve these systems. The Gibbs-Appell generalized method is an useful tool to determine the equations of motion of a system with constraints. In most cases, this method is more efficient to solve systems with linear non-holonomic constraints than the Lagrange multipliers (generalized) method or the resolution of the generalized Euler-Lagrange equations.

\section{References}

[1] L. Bates and J. Śniatycki, Nonholonomic reduction, Rep. Math. Phys. 32 (1993) 99-115.

[2] A. Cannas da Silva and A. Weinstein, Lectures on Geometrical Models for Noncommutative Algebra, University of California at Berkeley, 1998.

[3] J.F. Cariñena and Carlos López, Symplectic structure on the set of geodesics of a riemannian manifold, Int. J. Mod. Phys. A 6 (1991) 431-444.

[4] J.F. Cariñena and M.F. Rañada, Lagrangian systems with constraints: a geometric approach to the method of Lagrange multipliers, J. Phys. A: Math. Gen. 26 (1993) 1335-1351.

[5] J.F. Cariñena, J.M. Nunes da Costa, Patrícia Santos, Reduction of Lie algebroids structures, Int. J. Geom. Methods Mod. Phys. 2 (2005) 965-991.

[6] J.F. Cariñena, J.M. Nunes da Costa and Patrícia Santos, Reduction of Lagrangian mechanics on Lie algebroids, Submitted for publication. Preprint 05-29 Dep. Mat. Univ. Coimbra (http://www.mat.uc.pt/preprints/ps/p0529.pdf)

[7] J. Cortés and E. Martínez, Mechanical control systems on Lie algebroids, IMA J. Math. Control Inform. 21 (2004) 457-492.

[8] J. Cortés, M. de Léon, J.C. Marrero and E. Martínez, Nonholonomic Lagrangian systems on Lie algebroids, arXiv: math-ph/0512003.

[9] M. Crampin, On the differential geometry of the Euler-Lagrange equations, and the inverse problem of Lagrangian dynamics, J. Phys. A: Math. Gen. 14 (1981) 2567-2575.

[10] M. Crampin, Tangent bundle geometry for Lagrangian dynamics, J. Phys. A: Math. Gen. 16 (1983) 3755-3772.

[11] M. Crampin and F.A.E. Pirani, Applicable Differential Geometry, Cambridge U.P., 1986.

[12] E.A. Desloge, Classical Mechanics, Vol. 2, J. Wiley, New York, 1982.

[13] P.J. Higgins and K. Mackenzie, Algebraic constructions in the category of Lie algebroids, J. Algebra 129 (1990) 194-230.

[14] J. Koiller, Reduction of some classical non-holonomic systems with symmetry, Arch. Rational Mech. Anal. 118 (1991) 113-148.

[15] M. de León, J.C. Marrero and E. Martínez, Lagrangian submanifolds and dynamics on Lie algebroids, J. Phys. A: Math. Gen. 38 (2005) R241-R308. 
[16] A.D. Lewis, The geometry of the Gibbs-Appell equations and Gauss' principle of least constraint, Rep. Math. Phys. 38 (1996) 11-28.

[17] K. Mackenzie. Lie Groupoids and Lie Algebroids in Differential Geometry. London Math. Soc. Lect. Note Serie 124, Cambridge University Press, 1987.

[18] E. Martínez, Lagrangian mechanics on Lie algebroids, Acta Appl. Math. 67 (2001) 295-320.

[19] T. Mestdag and B. Langerock, A Lie algebroid framework for non-holonomic systems, J. Phys. A: Math. Gen. 38 (2005) 1097-1111.

[20] T. Mestdag, Lagrangian reduction by stages for non-holonomic systems in a Lie algebroid framework, J. Phys. A: Math. Gen. 38 (2005) 10157-10179.

[21] J.G. Papastavridis, Analytical Mechanics, Oxford University Press, 2002.

[22] J. Pradines, Théorie de Lie pour les groupoïdes différentiables. Calcul différentiel dans la catégorie des groupoïdes infinitésimaux, C.R. Acad. Sci. Paris Sér. A 264 (1967) 245-248.

[23] R. Rosenberg, Analytical Dynamics of Discrete Systems, Plenum Press, New York, 1977.

[24] A. Weinstein, Lagrangian mechanics and groupoids, in the book: Mechanics Day, Shadwick W.F. Krishnaprasad P.S. and Ratiu T.S. eds., Amer. Math. Soc., Fields Inst. Comm. 7 (1996) 207-231.

[25] E.T. Whittaker, A Treatise on the Analytical Dynamics of Particles and Rigid Bodies, Cambridge U.P., Cambridge, 1937.

J.F. CARIÑENA

Departamento de Física Teórica, Universidad de Zaragoza, 50009 Zaragoza, Spain

E-mail address: jfc@unizar.es

J.M. Nunes DA COSTA

Departamento de Matemática, Universidade de Coimbra, 3001-454 Coimbra, Portugal

E-mail address: jmcosta@mat.uc.pt

PATRÍ́cia SANTOS

Departamento de Física e Matemática, Instituto Superior de Engenharia de Coimbra 3030-199 Coimbra, Portugal

E-mail address: patricia@isec.pt 